\title{
Generalized Two-Dimensional Principal Component Analysis and Two Artificial Neural Networks to Detect Traveling lonospheric Disturbances Using the Tsunami-Atmosphere-Ionosphere Coupling Mechanism
}

Jyh-Woei Lin ( $\sim$ pgjwl1966@gmail.com )

Nanjing University of Information Science and Technology https://orcid.org/0000-0001-6875-0172

\section{Research Article}

Keywords: Bilateral Projection-Based Two-Dimensional Principal Component Analysis (B2DPCA), Traveling lonospheric Disturbance (TID), Back-Propagation Neural Network (BPNN), Convolutional Neural Network (CNN), BPNN-B2DPCA and CNN-B2DPCA methods

Posted Date: September 23rd, 2021

DOI: https://doi.org/10.21203/rs.3.rs-838410/v1

License: (c) (1) This work is licensed under a Creative Commons Attribution 4.0 International License. Read Full License 


\section{Generalized Two-Dimensional Principal Component Analysis and Two}

2 Artificial Neural Networks to Detect Traveling Ionospheric Disturbances

3 Using the Tsunami-Atmosphere-Ionosphere Coupling Mechanism

Jyh-Woei $\operatorname{Lin}^{12}$

1) Binjiang College, Nanjing University of Information Science \& Technology, Wuxi, 214105,

$$
\text { Jiangsu Province, China, pgjwl1966@gmail.com, }
$$

https://orcid.org/0000-0001-6875-01722), Tel: +8619906244722

2) Department of Electrical Engineering, Southern Taiwan University of Science and Technology, Tainan, Taiwan. Tel: +886966132209

Abstract: A weak tsunami was induced by the $2016 \mathrm{Mw}=7.8$ Sumatra earthquake, which occurred at 12:49 on March 2, 2016 (UTC). The epicenter was at $5.060^{\circ} \mathrm{S}, 94.170^{\circ} \mathrm{E}$ at a depth of $10 \mathrm{~km}$. At 15.02 on March 2 (UTC), the weak tsunami (amplitude: $0.11 \mathrm{~m}$ ) arrived at the station located at $10.40^{\circ} \mathrm{S}, 105.67^{\circ} \mathrm{E}$. The largest first principal eigenvalue derived using the bilateral projection-based two-dimensional principal component analysis (B2DPCA) indicated a spatial traveling ionospheric disturbance (TID), which was caused by internal gravity waves (IGWs), at 13:20 on March 2 (UTC). The largest second principal eigenvalue represented another TID expanding to the southwest. The two largest principal eigenvalues were associated with the TIDs, which were also determined using two back-propagation neural network (BPNN) models and two convolutional neural network (CNN) models, called the BPNN-B2DPCA and CNN-B2DPCA methods, respectively. These two methods yielded the same results as the B2DPCA. Therefore, the robustness and reliability of the B2DPCA were validated.

Keywords: Bilateral Projection-Based Two-Dimensional Principal Component Analysis (B2DPCA), Traveling Ionospheric Disturbance (TID), Back-Propagation Neural Network (BPNN), Convolutional Neural Network (CNN), BPNN-B2DPCA and CNN- B2DPCA methods

Correspondence Author:

Jyh-Woei Lin (pgjwl1966@gmail.com, (Dhttps://orcid.org/0000-0001-6875-01722) 
Spatial tsunami-associated ionospheric total electron content (TEC) anomalies caused by internal gravity waves (IGWs) are called spatial tsunami-associated ionospheric total electron content (TEC) anomalies. In this study, spatial tsunami-associated ionospheric total electron content (TEC) anomalies are named using traveling ionospheric disturbances (TIDs). They are explained by the concept of the tsunami-atmosphere-ionosphere (TAI) coupling mechanism (Hines, 1960; Hines, 1972; Molchanov and Hayakawa, 1998; Garcia et al., 2005; Shinagawa et al., 2007; Sun et al., 2007; Occhipinti et al., 2008; Rolland et al., 2010; Liu et al., 2011; Occhipinti et al., 2011; Occhipinti, 2015; Lean et al., 2016; Astafyeva, 2019). The unit of TEC is TECu, and 1 TECu $=10^{16} \mathrm{e} / \mathrm{m}^{2}$, where "e" and " $\mathrm{m}$ " represent electrons and meters, respectively (Hofmann-Wellenhof et al., 2001).

Contributing the concept of the TAI coupling mechanism, Hines (1960) observed irregularities and irregular motions in the upper atmosphere and found the first evidence of IGWs. Their propagation effects included amplification, reflection, intermodulation, and dissipation, which could reach ionospheric heights. Kherani et al. (2012) examined the Tohoku-Oki tsunami (March 11, 2011) using the TAI coupling mechanism concept through acoustic gravity waves (AGWs), IGWs, TEC data, and ground-based magnetometer measurements to observe the TIDs associated with the tsunami. The TIDs appeared as dipole-shaped disturbances near the epicenter, with the concentric circular wavefronts moving away radially from the epicenter with a horizontal velocity of approximately $800 \mathrm{~m} / \mathrm{s}$ after $12 \mathrm{~min}$. This was followed by a slow-moving (horizontal velocity $\sim 250 \mathrm{~m} / \mathrm{s}$ ) wave disturbance after $30 \mathrm{~min}$. Therefore, monitoring TIDs could serve as a real-time early warning of a tsunami. Garcia et al. (2014) described how a tsunami is coupled with ionospheric disturbances through IGWs. They used the gravity field and steady-state Ocean Circulation Explorer (GOCE) accelerometer to examine TEC data during the propagation of the tsunami caused by the Tohoku-Oki earthquake. Measurements of the air density and the vertical and horizontal velocities inside the IGWs were consistent with the responses generated by the tsunami. To estimate the propagation azimuth of the IGWs, researchers developed a new observation tool focused on the IGWs generated by tsunamis above the open ocean. Coïsson et al. (2015) analyzed the ionospheric data of radio occultation satellites to detect a tsunami-driven IGWs for the first time using a fully space-based ionospheric observation system combining TEC data of the Constellation Observing System for Meteorology, Ionosphere, and Climate (COSMIC) radio occultation measurements. After the 2011 Tohoku earthquake, the ionosphere was sounded from top to bottom, which provided the vertical structure of the IGWs excited by the tsunami propagation with the oscillations of the ionospheric TEC. The vertical wavelength was approximately $50 \mathrm{~km}$, and the maximum amplitude exceeded $1 \mathrm{TECu}$ when the occultation height reached $200 \mathrm{~km}$. Lin et al. (2015) developed a feasible, efficient, and low-cost tsunami early warning system in the South China Sea without the deployment of buoys. Lin (2015) used two-dimensional principal component analysis (2DPCA), which is a type of principal component analysis (PCA), to detect a TID after Japan's Tohoku earthquake that occurred at 05:46 on March 11, 2011 (UTC) $(\mathrm{Mw}=9)$. PCA is a linear method (Yang et al., 2004). Another TID near the epicenter occurred at 05:53. These TIDs were caused by the propagation of the tsunami. This method could serve as an early warning of tsunami because the speeds of the TIDs (3960-4950 km/h) were higher than those of the tsunami (720-800 $\mathrm{km} / \mathrm{h}$ ). However, the previous transform allowed for only the first principal eigenvalue to be assigned to the TIDs. All of the principal eigenvalues should be considered to detect more TIDs. Meng et al. (2015) found that the IGWs generated by tsunamis propagate upward, inducing TIDs in the ionosphere. They applied the global ionosphere-thermosphere model (GITM) to tsunami-generated IGWs to construct a three-dimensional physical model called wave perturbation-GITM (WP-GITM). The following tsunami wave properties were obtained: wave height, wave period, wavelength, and propagation direction. These were used as inputs for the WP-GITM. Combined with time-dependent characterization, the efficacy of this model was demonstrated through a simulation of the TEC variations near the West Coast of the United States around the time when they were reached by tsunami triggered by the March 2011 Tohuku earthquake. The simulated results of the TEC variations were consistent with global positioning system (GPS) observations. Consequently, in the WP-GITM, TIDs have been used as a signature of a tsunami.

Kamogawa et al. (2016) examined ionospheric plasma disturbances after a large tsunami using TEC data obtained from a GPS. TEC depressions corresponding to ionospheric holes formed over the tsunami areas in Japan and Chile. The largest TEC depressions appeared approximately 10-20 min after the earthquakes. Lean et al. (2016) developed a model that the spatial patterns of the ionospheric TEC response to solar, seasonal, diurnal, and geomagnetic influences could be determined across the globe using a new 
statistical model constructed from two-hourly TEC observations from 1998 to 2015 . The model combined physical solar photons and geomagnetic activity drivers with solar-modulated sinusoidal parameterizations of four seasonal cycles and solar- and seasonally modulated parameterizations of three diurnal cycles. Under this model, northern midlatitudes during winter were consistently under the influence of IGWs. Yu et al. (2017) found that ionospheric TEC fluctuations are affected by tsunamis through IGWs. A linear full-wave model and a nonlinear time-dependent model were used to examine the ionospheric responses to the IGW disturbances of the 2004 Sumatra and the 2011 Tohoku tsunamis. In the 2004 Sumatra tsunami, the IGW became unstable in the E layer at a distance of about $2000 \mathrm{~km}$ from the epicenter in one direction and $700 \mathrm{~km}$ in another one, thus limiting their detect ability at far distances. For the 2011 Tohoku tsunami, significant TIDs were observed moving northwest from the epicenter approximately $1 \mathrm{~h}$ following the tsunami. The IGWs propagating toward the southeast were responsible for the TIDs that propagated horizontally at almost $4000 \mathrm{~km}$. Astafyeva (2019) reported some natural hazards, e.g., earthquakes, tsunamis, and volcanic eruptions, that generated IGWs. They propagated upward and caused perturbations in the atmosphere and ionosphere. Liu et al. (2019) examined TIDs using the TEC data and ground-based GPS receivers. They further identified the TIDs in the 2004 Indian Ocean tsunami. Using the COMCOT model combined with analyses of the circle method, ray-tracing technique, and beam-forming technique, the TIDs were detected and confirmed immediately after the tsunami. Ravanelli et al. (2021) demonstrated a real-time tsunami genesis estimation using a GNSS and monitored the ionospheric TEC perturbations associated with the Mw 8.3 Illapel earthquake that occurred in Chile on September 16, 2015 (UTC). This method was based on the Total Variometric Approach (TVA) coupled with the Variometric Approach for Displacement Analysis Stand-Alone Engine (VADASE) with the VARION algorithm.

The objective of this study was to identify clear TIDs by the bilateral projection-based two-dimensional principal component analysis (B2DPCA) as a remote sensing tool using a tsunami record at a station. B2DPCA is a type of generalized two-dimensional PCA (Kong et al., 2005). Back-propagation neural networks (BPNN) and convolutional neural networks (CNN) were used to identify these clear TIDs. These analysis methods are called the BPNN-B2DPCA and CNN- B2DPCA methods, which were used to validate the robustness and the reliability of the B2DPCA.

B2DPCA is expected to potentially serve as a TID indicator without the complex modeling and complicated data processing involved in previous studies. For this study, a weak tsunami caused by the $\mathrm{Mw}=7.8$ Sumatra earthquake, which occurred at 12:49 on March 2, 2016 (UTC), with its epicenter at $5.060^{\circ} \mathrm{S}, 94.170^{\circ} \mathrm{E}$ at a depth of $10 \mathrm{~km}$ (Fig. 1), was selected for investigation. The earthquake that generated the weak tsunami was recorded using coastal sea-level gauges at several stations in the Indian Ocean, namely, those in Australia (Cocos and Christmas Islands), Indonesia (Tanahbala), Maldives (Gan, Male, and Hanimadhoo), and Mauritius (Rodrigues Island). The estimated amplitude of the tsunami was $0.11 \mathrm{~m}$ (peak value) around Christmas Island (International Tsunami Information Center, ITIC; Indian Tsunami Early Warning Centre, ITEWC). A $0.11 \mathrm{~m}$ tsunami is significant in the open ocean but moderate around coastal regions (Okal and Synolakis, 2016; Sim and Huang, 2016; Sun et al., 2018; Heidarzadeh et al., 2019). The measured wave amplitude was not a simulated value, but it was determined from collected data. All of the principal eigenvalues were examined to detect all of the possible TIDs. B2DPCA is a nonlinear method capable of detecting nonlinear ionospheric TEC variations (Kong et al., 2005). At 15:02 on March 2, 2016 (UTC), a weak tsunami arrived at the station near Christmas Island at $10.40^{\circ} \mathrm{S}, 105.67^{\circ} \mathrm{E}$. The TIDs associated with this tsunami were detected from 12:50 to 15:00. These TIDs were analyzed in this study. The TEC data from 12:50 to $15: 00$ were selected because the epicenter $\left(5.060^{\circ} \mathrm{S}, 94.170^{\circ} \mathrm{E}\right)$ was far from the coast of Christmas Island, and thus, it was a weak tsunami when it arrived at this station (Fig. 1). The TIDs were detected using B2DPCA, which demonstrated the high sensitivity of this method in detecting TEC anomalies related to a weak tsunami (a $0.11 \mathrm{~m}$ tsunami) for the selected time range of the TEC data. The Indonesian and Australian authorities called off their tsunami alerts within $2 \mathrm{~h}$ of the tremors. The Indonesian Meteorology and Geophysics Agency declared the likelihood of a tsunami to be very weak. Therefore, while no clear tsunami was seen at the southwest coast of Sumatra, Indonesia, its arrival at this station could be used as a reference because the arrival time was known.

\section{SOURCE DATA}

The Global Differential GPS (GDGPS) system offers complete, highly accurate, and extremely robust real-time GPS monitoring. This system provides decimeter-level positioning accuracy and produces two-dimensional TEC values with a global spatial resolution of the latitude and longitude of $2.5^{\circ}$ and $5^{\circ}$, respectively (Hernández-Pajares et al., 2009; Guo et al., 2015; Ren et al., 2016). The spatial resolution was 
very low. Therefore, they belong to the sparse spatial sampling datasets called the small sample size (SSS) datasets (Golugula et al., 2011). The temporal resolution of the global TEC data was 5 min. More detailed information, system architecture and operating concept about the GDGPS system can be found at https://iono.jpl.nasa.gov/latest_rti_global.html and in several previous publications (Bertiger et al., 1997; Hernández-Pajares et al., 2009; Guo et al., 2015; Ren et al., 2016; Shi and Wei, 2020).

\section{METHOD}

B2DPCA is a nonlinear method. The input matrix is denoted as $\mathrm{W}_{I}$ with the dimensions $n \times m$. A linear projection is represented as follows (Yang et al., 2004; Kong et al., 2005):

$$
B_{I}=\mathrm{U}\left(W_{I}\right) \mathrm{V}
$$

where $\mathrm{U}$ and $\mathrm{V}$ are two multiplying projection matrices.

$B_{I}$ is the extracted feature matrix, which is mapped using $\mathrm{U}$ :

$\mathrm{M}=\sum_{i=1}^{n \times m}\left\|W_{I}-U B_{I} V^{T}\right\|$

where $\|\bullet\|$ represents the Frobenius norm of a matrix, and $n \times m$ is the number of elements for the input matrix. The following equation is used to maximize Equation (2):

$\mathrm{J}=\sum_{i=1}^{n \times m}\left\{\operatorname{tr}\left(B_{I} B_{I}{ }^{T}\right)-\operatorname{tr}\left(B_{I} W_{I}\right)\right\}$

yielding the principal eigenvalue of $\mathrm{B}_{I}$. The letters " $\mathrm{T}$ " indicate the transpose of a matrix. The letters " $\operatorname{tr}$ " indicate the trace of a matrix. Thus, this results in the total number of principal eigenvalues being $I$, which are denoted as $\lambda_{1}, \lambda_{2} \ldots \lambda_{I}$, corresponding to the eigenvectors $w_{1}, w_{2} \ldots w_{I}$. Each principal eigenvalue corresponds to an eigenvector and represents part of the dataset characteristics. Therefore, all of the characteristics of this dataset can be represented using the principal eigenvalues from largest to smallest. In this study, the principal eigenvalue with the smallest magnitude is represented as $\lambda_{1}$, and that with the largest magnitude is represented as $\lambda_{\mathrm{I}}$. Thus, $\lambda_{1}>\lambda_{2}>\ldots>\lambda_{I}$. These principal eigenvalues indicate all of the characteristics of these data by transforming B2DPCA. The previous operation can be treated as a domain transformation, meaning that the dataset is transformed into another domain, with the bases as eigenvectors. This is the mathematical and physical meaning of B2DPCA. B2DPCA can encode the entire sparse spatial sampling datasets e.g., the TEC data of the GDGPS system in this study, with the input matrix, which is the SSS datasets, thereby minimizing the loss of information of such dataset (Golugula et al., 2011).

\section{DATA PROCESSING AND RESULTS OF B2DPCA}

The global TEC data that were collected every 5 min from 12:50 to 15:00 on March 2, 2016 (UTC) were processed using B2DPCA to identify TIDs, which were represented by relatively large principal eigenvalues. No other TIDs were detected using B2DPCA at any of the surrounding stations during this period. It was expected that all TIDs from 12:50 to 15:00 could be traced through examination of all of the global TEC datasets. This study focused on the TEC data collected from 12:50 to 15:00 to demonstrate the sensitivity of the B2DPCA in detecting TIDs.

Fig. 2 presents the processed global TEC data, where the Global ionospheric TEC map (GIM) at 13:20 on March 2, 2016 (UTC) was divided into 600 grids with latitude and longitude resolutions of $9^{\circ}$ and $12^{\circ}$, respectively. As expressed in Equation (1), the TEC dataset in each grid, which was SSS dataset (four TEC data in each grid), were used to form a $2 \times 2$ input matrix $\mathrm{W}_{I}$ (spatial resolution: latitude $\times$ longitude $=$ $2.5^{\circ} \times 5^{\circ}$ for TEC data). The TEC datasets were processed according to the method stated in Section III. The B2DPCA can deal with the SSS datasets. An input matrix contained SSS dataset for each grid (600 grids), and thus, the spatial structure and information of the datasets were well preserved, resulting in two principal eigenvalues: $\lambda_{1}>\lambda_{2}$. Therefore, 600 first and 600 second principal eigenvalues of the B2DPCA were estimated. For comparison, all of the principal eigenvalues of the B2DPCA and PCA are presented in Fig. 3 and 4, respectively. Fig. 3 (a) and (b) use a color-coded scale of the magnitudes of the first and second principal eigenvalues corresponding to data in Fig. 2. The color intensity denotes the magnitude. Fig. 4 (a) and (b) use a color-coded scale of the magnitudes of the principal eigenvalues corresponding to data in Fig. 2 acquired using PCA. To validate the robustness and reliability of the B2DPCA, the two TIDs 
were also determined using two BPNN models and two CNN models. The validation procedures are introduced in the following sections.

\section{VALIDATION BY TWO BACK-PROPAGATION NEURAL NETWORK (BPNN) MODELS}

Two BPNN models were constructed to predict the magnitudes of all of the principal eigenvalues of the B2DPCA to determine whether the largest first and second principal eigenvalues were associated with the TIDs. Detailed explanations of BPNNs are available in the publications of Lin et al. (2018) and Lin and Chiou (2019). The BPNN models were constructed as follows:

(1) The BPNN framework had two hidden layers and 10 neurons in each hidden layer (Fig. 5), where $\mathrm{J}=\mathrm{K}$ $=10$. Some researchers have indicated that a neural network with two hidden layers and few neurons can replace a network with numerous neurons in one hidden layer (Lin et al., 2018; Lin and Chiou, 2019; Chu et al., 2020). The training data were used to train the BPNN. In neural networks, a training dataset consists of labeled data (Belo et al., 2017) that includes training inputs and target outputs, which were real values in this study (Tuntisukrarom and Cheerarot, 2020). The TEC data were transformed into (i.e., mapped to) the principal eigenvalues (using the concept of mapping the TEC data from one domain to another domain. i.e., mapping from the TEC domain to the principal eigenvalue domain).

(2) For the first BPNN model, all the first principal eigenvalues of the B2DPCA (Fig. 3a), with random noise added in the range of $0-0.5$ (Chen et al., 2013) as target outputs, and their corresponding grids

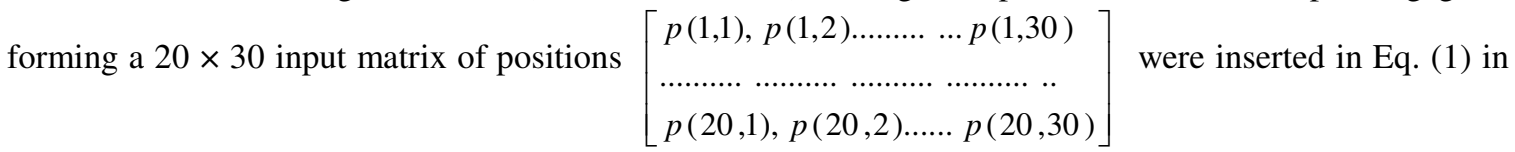

the studies of Lin et al. (2018) and Lin \& Chiou (2019) as the training inputs to form the training data. The largest principal eigenvalues were considered to indicate the TIDs. The concept was similar to that of other studies. Thus, the predicted results were expected to have high accuracy, especially for training data with small magnitudes (Harbaugh et al., 2000; Chen et al., 2013; Eslamian, 2014; Mohamed, 2019; Dao et al., 2020). In this study, the principal eigenvalues were extremely small, thus necessitating the addition of random noise to enhance the prediction accuracy. Chen et al. (2013) selected pumping rates by adding a random value in the range of $0-0.5 \mathrm{~cm} / \mathrm{s}$ as a training input in an area of $100 \times 100 \mathrm{~m}^{2}$, which was divided into $17 \times 17 \mathrm{~m}^{2}$ grids for the conjunctive use of surface and subsurface water for two-dimensional groundwater using BPNN modelling due to the low pumping rates as training inputs. Therefore, using the predicted pumping rate BPNN model, a more accurate groundwater simulation was achieved, which was valuable for applications.

(3) The Levenberg-Marquardt algorithm (LMA) (Haykin, 2008) served as the EBP algorithm of the BPNN model. The training inputs were normalized in the range of $0-1$ with feature scaling (Bo et al., 2006). The sigmoid function was used as an activation function (Haykin, 2008).

(4) The initial weights and the initial biases were set to random variables between 0 and 1 because this is the range of the sigmoid function (Haykin, 2008) that was used in this study. The training error was defined as the target output minus the temporary output in each epoch of the training process using the EBP algorithm (Omatu et al., 2018). The predicted error was defined as the predicted value minus the target output.

(5) The number of training epochs was set to 2000, and the learning rate was adaptive at 0 to 1 , with an increment of 0.01 . The best learning rate of 0.67 , which yielded minimal training error, was used to build the first BPNN model to predict the second principal eigenvalues for the B2DPCA in Fig. 3b. When the number of training epochs was set to more than 2000, overfitting (Chicco, 2017) occurred.

(6) To verify the reliable application of the first model, the first principal eigenvalues, which were obtained from the training data, were predicted by the first BPNN model, and the results are presented in Fig. 6 (a). This means that the training data were used to validate the reliability and robustness of the model (inside test). To evaluate the reliable application of the first BPNN, the second principal eigenvalues, which were not obtained from the training data, were predicted by the first BPNN model, and the results are presented in Fig. 6 (b) (outside test). Thus, data that were not training data were used to test the reliable application of 
the model (outside test). As stated previously, this concept was derived from Bhadra et al. (2017) and Bo et al. (2006), and it is a type of cross-validation (inside and outside tests) of the model.

(7) The standard deviation (STD) and mean square error (MSE) (Maleki and Zabihollah, 2016) were used as statistical approaches to evaluate the reliable application and predicted accuracy of the first BPNN model. The STD and MSE values of the predictions using the first BPNN model were 0.012 and 0.020 in the inside test, respectively, as shown in Fig. 6(a). The STD and MSE values of the predictions using the first BPNN model were 0.032 and 0.043 in the outside test in Fig. 6(b). This was a type of cross-validation of the first BPNN model. Therefore, the low STD and MSE values obtained by the first BPNN model indicated a certain reliability of the B2DPCA.

(8) Using the same training and analysis method as stated above, all of the second principal eigenvalues of the B2DPCA in Fig. 3b, in which random noise was added in the range $0-0.5$ and corresponding grids formed a $20 \times 30$ matrix of positions, were used as the training data to construct the second BPNN model. The same procedure as that used to build the first BPNN model was performed to construct a second BPNN model. The best learning rate was 0.48 , and the training error for the predictions of the principal eigenvalues of the B2DPCA was minimal, as depicted in Fig. 7. To evaluate the prediction accuracy of the second BPNN, the first principal eigenvalues, which were not obtained using training data, were predicted by the second BPNN model, as presented in Fig. 7 (a) (outside test). To verify the reliable application of the second BPNN model, the second principal eigenvalues, which were obtained using the training data, were predicted by the second BPNN model presented in Fig. 7 (b) (inside test). The STD and MSE values of the predictions using the second BPNN model were 0.021 and 0.034, respectively, as shown in Fig 7 (a). The STD and MSE values of the predicted errors using the second BPNN model were 0.012 and 0.022 , respectively, as shown in Fig 7 (b). This served as a type of cross-validation of the second BPNN model. Therefore, the low STD and MSE values obtained by the second BPNN model indicated a certain reliability of the B2DPCA.

Fig. 3 (a) and (b) are similar to Fig. 6 (a) and (b), respectively, and to Fig. 7 (a) and (b), respectively. The cross-validation results demonstrated the applicability of both BPNN models. Both principal eigenvalues corresponded to TIDs. The flowchart for creating the two BPNN models is displayed in Fig. 8 . As stated previously, the TEC data were transformed into the principal eigenvalues. The BPNN confirmed the distribution of the principal eigenvalues of the B2DPCA. This procedure is called the BPNN-B2DPCA method.

\section{VALIDATION BY TWO CONVOLUTIONAL NEURAL NETWORK (CNN) MODELS}

Two CNN models were built to classify TIDs and non-TIDs by splitting the TEC map in Fig. 2 into 600 images. Two images corresponding to the largest first and second principal eigenvalues of Fig. 3 were supposed to be TIDs. The framework of a two-dimensional CNN with two hidden layers is displayed in Fig. 9. The procedure and concept for creating TEC images using two CNN models were similar to the procedure presented by Lin \& Chiou (2020). Therefore, image processing was necessary before training the CNN models. A detailed explanation of CNNs is available in the publication of Lin \& Chiou (2020).

In this study, the $\mathrm{CNN}$ models were constructed as follows:

(1) To build the first CNN model, the TEC map in Fig. 2 was split into 600 images. The size of each training image was $9^{\circ}$ in latitude and $12^{\circ}$ in longitude. The scales of the horizontal and vertical axes were the same as those in Fig. 3. Some of the 600 images will be used as the training images for Eq. 1 in the study of Lin \& Chiou (2020).

(2) The term "training images" was defined as the training input. The target outputs of the two CNN models were defined as the similarities of the images, with values between 0 and 1 . When similarity $=0$, the output was classified as the first classification, with no information on the TIDs in the images. When similarity $=1$, the output was classified as the second classification with full information on the TIDs in the images. However, in a real situation, the similarity is never 0 or 1 (Lin \& Chiou, 2020).

(3) According to the study of Lin \& Chiou (2020), a threshold of the similarities with 0.5 was determined. The similarities of $0-0.5$ were defined as the first classification, which indicated a non-TID. The 
similarities of $0.5-1$ were defined as the second classification, which indicated a TID. A similarity of exactly 0.5 was deemed meaningless (Lin \& Chiou, 2020). (3) After splitting the TEC map, the lateral sides of the 600 training images contained a type of artificial image processing noise called wedge effects in image processing because they contained discontinuities (Baert et al., 2014; Lin \& Chiou, 2020). A low-pass Butterworth filter (Bianchi and Sorrentino, 2007) was used to reduce the wedge effects for each training image before training the CNN model, because the Butterworth filter was identified as a suitable filter for TEC data and TEC images (Chandrasekhar et al., 2016; Lin \& Chiou, 2020).

(4) A low-pass Butterworth filter was also used to remove black curves and lines that indicated the contours of the land and lines that indicated the scales of the latitude and longitude for the TEC map in Fig. 2. Some training images included these curves and lines. Therefore, a new image processing procedure was designed. These curves and lines could be treated as discontinuities in these images (artificial image noises), and the discontinuities caused the wedge effects during training of the CNN. Thus, the low-pass Butterworth filter was used. A training image containing curves and lines was split along these curves and lines into subimages. The low-pass Butterworth filter was then used to remove the wedge effects of these subimages. After removing these effects, these subimages were reassembled into an image as a reconstructed training image like a puzzle. Some studies have used similar image processing methods (e.g., Koundal and Gupta, 2020), but the process used was not exactly the same as the process described above. Thus, this is an important new processing method.

(5) The initial weights and biases were set to random variables (Nguyen and Widrow, 2009). The number of training epochs was set as 2000 (Omatu et al., 2018), and the learning rate was adaptive in the range from 0 to 1 , with an increment of 0.01 . When the number of training epochs was set to more than 2000, overfitting occurred. The batch size was set to 3 to reduce the computing time (Palm, 2012; Lin \& Chiou, 2020). Feature scaling was then performed to ensure that these variables were between 0 and 1 (Bo et al., 2006), because the range of the sigmoid function was 0-1. Simultaneously, batch normalization was performed as described above. The LMA algorithm, as the EBP algorithm, was used to obtain optimal weights.

(6) The split images on the left side of the $96^{\circ}$ longitude $\left({ }^{\circ} \mathrm{E}\right)$ of an image, corresponding to the largest first principal eigenvalues of Fig. 3(a), were used as training inputs (461 images) to build the first CNN model. This model was used to classify 600 images, which were classified as the first or second classification based on the similarity values.

(7) The best learning rate of the first CNN model with minimal training error was 0.36 . A training error was defined as the target output minus the temporary output in each epoch of the training process using the EBP algorithm (Omatu et al., 2018). A prediction error was defined as the predicted value minus the target output.

(8) The classification results are displayed in Fig. 10 for the evaluation of the reliability and prediction accuracy of the first CNN model. The evaluation of the reliability revealed that the predicted errors of similarities using this CNN model were to the left of $96^{\circ}$ longitude $\left({ }^{\circ} \mathrm{E}\right)$, with an STD and MSE (inside test) of 0.21 and 0.25 , respectively. The evaluation of the prediction accuracy revealed that the predicted errors of similarities were to the right of $96^{\circ}$ longitude $\left({ }^{\circ} \mathrm{E}\right.$ ), with an STD and MSE (outside test) of 0.28 and 0.31 , respectively. Low STD and MSE values verified that the first CNN model was reliable. This served as a type of cross-validation of the first CNN model.

(9) Using the same concept as that used to build the first CNN model, to build the second CNN model, split images with the areas on the left side of the $84^{\circ}$ longitude $\left({ }^{\circ} \mathrm{E}\right)$ for an image corresponding to the largest second principal eigenvalues in Fig. 3(b) were used as training inputs (441 images) to build the second CNN model. This CNN was used to classify 600 split images based on the values for grids with first principal eigenvalues corresponding to Fig. 3 obtained using B2DPCA. The best learning rate of the second CNN model with minimal training error was 0.28 . The classification results are displayed in Fig. 11, and the results show the reliability and prediction accuracy of the second CNN model. The evaluation of the reliability revealed that the predicted errors of similarities using this CNN model were to the left of $84^{\circ}$ longitude $\left({ }^{\circ} \mathrm{E}\right)$, with an STD and MSE (inside test) of 0.26 and 0.27 , respectively. The evaluation of prediction accuracy revealed that the predicted errors of the similarities were to the right of $84^{\circ}$ longitude $\left({ }^{\circ} \mathrm{E}\right)$, with an STD and MSE (outside test) of 0.32 and 0.35, respectively. Low STD and MSE values verified that the second CNN model was reliable. This served as cross-validation of the second CNN model. 
(10) The images in Fig. 10 and 11 were classified as the second classification (similarities $>0.5$ ), and they were located at the same positions as the largest principal eigenvalues, as depicted in Fig. 3 (a) and (b). Therefore, these two images were considered to represent a TID. The flowchart for creating the two CNN models is illustrated in Fig. 12. The CNN confirmed the TEC variants by classifying 600 split images. Therefore, the TIDs can be indirectly recognized through a CNN, which is called the CNN-B2DPCA method.

\section{DISCUSSION}

Typically, the training dataset of an artificial neural network (ANN) is used to validate the reliability and robustness of this model, called the inside test. An additional dataset, i.e., the test dataset, is used to test the prediction ability of this model, called outside test. This is a type of cross-validation (Bo et al., 2006; Bhadra et al., 2017). The training dataset in this study was a small dataset. Performing cross-validation is an important issue to maintain a better prediction accuracy of this ANN model and avoid overfitting (Pasini, 2015; Shaikhina and Khovanova, 2017; Baquirin and Fernandez, 2018; Seddiki et al., 2020; Zakeri et al., 2020; Hemmerich et al., 2020). An ANN model shows an accurate prediction ability through cross-validation. For a high-quality prediction model, the prediction accuracies of the inside tests should be better than those of outside tests (Bo, et al., 2006; Bhadra et al., 2017). The previous STD and MSE values of the inside and outside tests validated the reliability, robustness, and accuracy of the two BPNN and two CNN models. The two largest principal eigenvalues were detected using B2DPCA at 13:20 and were treated as an indicator of TIDs. However, in this example, PCA did not detect any TIDs. The BPNN-B2DPCA and CNN-B2DPCA methods were used to verify that the two largest principal eigenvalues were the two TIDs.

Associated with a weak tsunami and the concept of the TAI coupling mechanism, the TIDs were created through the IGWs (Liu et al., 2011). Liu et al. (2011) identified TEC anomalies caused by IGWs that resulted from the tsunami caused by the March 11, 2011, Tohoku earthquake. B2DPCA offers a mathematical tool for detecting TIDs, which may help characterize their source. At 15.02 on March 2, 2016 (UTC), a $0.11 \mathrm{~m}$ tsunami arrived at $10.40^{\circ} \mathrm{S}, 105.67^{\circ} \mathrm{E}$ and caused a TID, as determined from the characteristics of the first largest principal eigenvalue when using B2DPCA at 13:20 on March 2, 2016 (UTC). Simultaneously, a second TID represented by the second largest principal eigenvalue that was expanding to the southwest was detected. The predicted results of the two BPNN and CNN models (the BPNN-B2DPCA and CNN-B2DPCA methods, respectively) validated that the largest principal eigenvalues were associated with the TIDs. Therefore, the results of this study indicated that a new method for detecting tsunamis is available because all of the methods produced the same results. The two TIDs represented by the two largest principal eigenvalues (13:20) were detected approximately $100 \mathrm{~min}$ prior to the tsunami at the station (15.02). This may be attributable to the depth and focal mechanism of an earthquake, as the amplitude of a tsunami is determined by the magnitude of the displacement of the sea floor (Kawashima and Toh, 2016; Heidarzadeh et al., 2017; Pennin, 2018; Widiyantoro et al., 2020). This earthquake could not induce large turbulence in the sea, so the amplitude of the tsunami had no information around the epicenter. Later, the amplitude of the tsunami was small when passing through the deep sea, which may have prevented the detection of more spatial and temporal TIDs by B2DPCA. Therefore, TIDs were not found at other time points (incl. time point close to occurred time of this earthquake), so that the speeds of the TIDs could not be estimated. Additionally, the temporal resolution of the TEC data for GDGPS system was $5 \mathrm{~min}$. Increasing the spatial and temporal sampling rate could allow for detection of more spatial and temporal TIDs by B2DPCA. Notably, the results in Fig. 10 and 11 indicated that the removal effects using the Butterworth filter were good for the black curves and lines, which indicated the contours of the land and the scales of the latitude and longitude, respectively, as stated previously. This study was the first to use this approach.

It is worth mentioning that the Sumatra earthquake occurred at 12:49 and the TEC data in the time period from 12:50 to 15.00 were examined for detecting TIDs using B2DPCA. However, only two TIDs at 13:20 were detectable. In this case, this weak tsunami did not produce any TIDs at other time points using B2DPCA. The two TIDs were also detected using the BPNN-B2DPCA and CNN-B2DPCA methods. However, the results of this study achieved the objective.

The properties of the propagation of IGWs in the atmosphere are related, and IGWs interact with the ionospheric plasma, which is the coupling strength between the geomagnetic field and ionospheric plasma. 
This coupling strength determines the possibility of the propagation of the IGWs reaching the ionosphere height (Occhipinti et al., 2008). The problem of the propagation of IGWs of tsunamis is also based on boundary conditions of explicit formulas that relate the pressure, density, and velocity at the interfaces between water, air, and gas layers. These boundary conditions characterize the IGWs, which reach an arbitrary height, including the height of the ionosphere. Under the condition that the boundary conditions are not satisfied, the propagation of IGWs may not reach the ionosphere height to produce TIDs (Leble and Smirnova, 2019). The IGWs are accompanied by the electrical current generated by a tsunami reaching the ionosphere height to produce TIDs. In the ocean, the electric current is generated by the motion of seawater in the geomagnetic field (Lilley et al., 2001; Šachl, et al., 2019; Xu et al., 2018; Minami et al., 2021). The electric current can perturb the geomagnetic field. The electric current variation is an important issue. Therefore, this variation, which is a boundary condition, determines the propagation of IGWs to produce TIDs at the ionosphere height (Sorokin and Yaschenko, 2021).

As stated previously, the two TIDs were only detected at 13:20. At other time points (incl. time point close to occurred time of this earthquake), this tsunami may cause IGWs. However, the characteristics of IGWs do not satisfy the coupling strength between the geomagnetic field and ionospheric plasma (e.g. weak coupling strength), the boundary conditions of explicit formulas and the boundary condition of the electric current variation (e.g. weak electric current) (Occhipinti et al., 2008; Leble and Smirnova, 2019; Sorokin and Yaschenko, 2021), so that corresponding IGWs could not reach ionospheric heights to produce TIDs at other time points.

A comparison with the results of previous studies is presented. Aside from the study of Lin (2015), other previous studies indicated that TIDs are affected by space weather, and complex models and complicated data processing were necessary. However, only the first large principal eigenvalue of 2DPCA was used to detect the TIDs. Other large principal eigenvalues were not examined. Therefore, some TIDs may be not found by the analysis of Lin (2015). All of the principal eigenvalues of the B2DPCA were used to avoid information loss and to detect two TIDs. In this study, the selected tsunami was weak. However, when a tsunami is stark and clear, clear TIDs should be simultaneously detected by the B2DPCA after extending the time range of the TEC data. Consequently, B2DPCA can be a powerful and significant tool to detect clear TIDs. Furthermore, use of the B2DPCA does not require use of a simulation model or a shake map associated with a tsunami; it only uses data collected after the event.

The observation methods on which the past studies relied were subjective. A mathematical tool such as B2DPCA for the computation of TIDs, which can be applied without complex models and complicated data processing, will be valuable because it is more objective and sensitive than other approaches. The TIDs were identified by examining the disturbance storm time (Dst) indices in Fig. 13 for March 2, 2016. The Dst indices indicate part of the space weather (Hamilton et al., 1988). The Dst indices indicate the variations of a certain degree on March 2, 2016. Therefore, the processing of B2DPCA was independent of the variations of the Dst indices. That is, if space weather and other irregularities or anomalies in the ionosphere affect the results of the B2DPCA, then anomalous principal eigenvalues should exist in other grids. The characteristics of the two TIDs could be regarded as the nonlinear expansion of plasma that was detected using B2DPCA. This type of nonlinear analysis is an advantage of B2DPCA. The two TIDs could not be identified using PCA. Therefore, B2DPCA solved the problem of identifying TIDs. One limitation of this study, as stated previously, is the lack of detection of other TIDs using B2DPCA at other time points. If more TIDs can be detected, combining these TIDs, then the speeds of the TIDs can be calculated. Thus, a real-time early warning of tsunamis for surrounding regions (Lin, 2015) can be developed using the methods in this study. This will be a subject of future research.

\section{CONCLUSION}

B2DPCA was used to identify two TIDs induced by IGWs. Two TIDs appeared at 13:20 on March 2, 2016 (UTC), accompanying the Sumatra earthquake that occurred at 12:49 on March 2, 2016 (UTC). The epicenter of the earthquake was located at $5.060^{\circ} \mathrm{S}, 94.170^{\circ} \mathrm{E}$ at a depth of $10 \mathrm{~km}$. The two TIDs appeared approximately 100 min prior to the arrival of the tsunami at this station. Simultaneously, another TID expanded to the southwest. This weak tsunami did not produce any TIDs at other time points using B2DPCA. These results were not affected by space weather and other irregularities or anomalies in the ionosphere. The two TIDs were caused by the TAI coupling mechanism, which was not detected by PCA. To validate the robustness and reliability of the B2DPCA, the two TIDs were also determined using two BPNN models and two CNN models, which were called the BPNN-B2DPCA and CNN-B2DPCA 
methods, respectively. The reliability of the two methods was validated, and the B2DPCA yielded low STD and MSE values. Furthermore, these two methods yielded the same results as the B2DPCA.

Acknowledgements

470

The author is grateful to the NASA Global Differential GPS system (GDGPS) and World Data Centre for

Data Availability

476

The TEC data can be obtained in NASA Global Differential GPS system (GDGPS)

477 MATLAB code source The author modified the code for the objectives of this study. Conflicts of Interest: The author declares that there are no conflicts of interest. 
References:

Astafyeva, E., 2019, Ionospheric Detection of Natural Hazards, Reviews of Geophysics, Vol. 57(4), 1265-1288, doi: 10.1029/2019RG000668.

Baert, I. A. C., Nijs, J., Meeus, M., Lluch, E. and Struyf, F., 2014, The effect of lateral wedge insoles in patients with medial compartment knee osteoarthritis: balancing biomechanics with pain neuroscience, Clinical Rheumatology Vol. 33, 1529-1538, doi: 10.1007/s10067-014-2668-1

Baquirin, R. B. M. and Fernandez, P. L., 2018, Artificial Neural Network (ANN) in a Small Dataset to determine Neutrality in the Pronunciation of English as a Foreign Language in Filipino Call Center Agents, Inteligencia Artificial, Vol.21(62), 134-144, doi: 10.4114/intartif.vol21iss62pp134-144

Belo, D, Rodrigues, J., Vaz, J. R., Pezarar-Correia, P. and Gamboa, H., 2017, Biosignals learning and synthesis using deep neural networks, BioMedical Engineering OnLine Vol. 16, 115, doi: 10.1186/s12938-017-0405-0

Bertiger, W. I., Bar-Sever, Y.E., Haines, B. J., Iijima, B. A., Lichten, S. M., Lindqwister, U. J., Mannucci, A. J., Muellerschoen, R.J., Munson, T. N., Moore, A.W., Romans, L. J., Wilson, B. D., Wu, S. C., Yunck, T. P., Piesinger, G. and Whitehe, M., 1997, A Real-Time Wide Area Differential GPS System, Navigation, Vol.44 (4), 433-447, doi: 10.1002/j.2161-4296.1997.tb02359.x

Bhadra, A., Bandyopadhyay, A., Clakraborty, S. and Kumar, T., 2017, Development and Testing of an ANN Model for Estimation of Runoff from a Snow Covered Catchment, Journal of The Institution of Engineers (India): Series A, Vol. 98, 29-39, doi: 10.1007/s40030-017-0203-5

Bianchi, G. and Sorrentino, R., 2007, Electronic Filter Simulation \& Design, McGraw Hill Professional, pp.606, ISBN: 9780071494670

Bo, L., Wang, L. and Jiao, L., 2006, Feature scaling for Kernel Fisher discriminant analysis using leave-one-out cross validation. Neural Computation, Vol.18 (4), 961-978, doi: 10.1162/neco.2006.18.4.961

Chandrasekhar, E., Prabhudesai, S. S., Seemala, G. K. and Shenvi, N., 2016, Multifractal detrended fluctuation analysis of ionospheric total electron content data during solar minimum and maximum, Journal of Atmospheric and Solar-Terrestrial Physics, Vol. 149, 31-39, doi: 10.1016/j.jastp.2016.09.007

Chen, Y. W., L. C. Chang, C. W. Huang and H. J. Chu, 2013, Applying Genetic Algorithm and Neural Network to the Conjunctive Use of Surface and Subsurface Water, Water Resour Manage, 27, 4731-4757, doi:10.1007/s11269-013-0418-9

Chicco, D., 2017, Ten quick tips for machine learning in computational biology, BioData Mining, 10, 35, doi: 10.1186/s13040-017-0155-3

Chu, Y., Fei, J. and Hou, S., 2020, Adaptive Global Sliding-Mode Control for Dynamic Systems Using Double Hidden Layer Recurrent Neural Network Structure, IEEE Transactions on Neural Networks and Learning Systems, Vol. 31(4), 1297 - 1309, doi:10.1109/TNNLS.2019.2919676

Coïsson, P., Lognonné, P., Walwer, D., and Rolland, L. M., 2015, First tsunami gravity wave detection in ionospheric radio occultation data, Earth and Space Science, Vol.2(5), 125-133, doi:10.1002/2014EA000054

Dao, D. V., Adeli, H., Ly, H. B., Le, L. M., Le, V. M., Le, T. T. and Pham, B. T., 2020, A Sensitivity and Robustness Analysis of GPR and ANN for High-Performance Concrete Compressive Strength Prediction Using a Monte Carlo Simulation, Sustainability, Vol. 12 (3), 830, doi:10.3390/su12030830

Eslamian, S., 2014, Handbook of Engineering Hydrology: Modeling, Climate Change, and Variability, pp.646, Handbook of Engineering Hydrology, Vol.2, CRC Press, ISBN: 9781466552470

Freund, F. T., 2003, Rocks That Crackle and Sparkle and Glow Strange Pre-Earthquake Phenomena. Journal of Scientific Exploration, Vol. 17, No. 1, 37-71.

Golugula, A., Lee, G. and Madabhushi, A., 2011, Evaluating feature selection strategies for high dimensional, small sample size datasets, 2011 Annual International Conference of the IEEE Engineering in Medicine and Biology Society, PubMed ID: 22254468, doi: 10.1109/IEMBS.2011.6090214

Garcia, R., Crespon, F., Ducic, V. and Lognonné, P., 2005, Three-dimensional ionospheric tomography of post-seismic disturbances produced by the Denali earthquake from GPS data. Geophysical Journal International, Vol.163 (3), 1049-1064, doi: 10.1111/j.1365-246X.2005.02775.x

Garcia, R. F., Doornbos, E., Bruinsma, S. and Hebert, H., 2014, Atmospheric gravity waves due to the Tohoku-Oki tsunami observed in the thermosphere by GOCE, Journal of Geophysical Research, 
Atmospheres, Vol.119 (8), 4498-4506, doi: 10.1002/2013JD021120

Guo, J., Li, W., Liu, X., Kong, Q., Zhao, C. and Guo, B, 2015, Temporal-Spatial Variation of Global GPS-Derived Total Electron Content, 1999-2013, PLoS ONE, Vol. 10(7), e0133378. doi:10.1371/journal.pone.0133378

Hamilton, D. C., Gloeckler, G., Ipavich, F. M., Studemann, W., Wilken, B. and Kremser, G., 1988, Ring current development during the great geomagnetic storm of February, Journal of Geophysical Research, Space Physics, Vol.93 (A12), 14343-14355, doi: 10.1029/JA093iA12p14343

Harbaugh, A. W.., E. R. Banta., M. C. Hill and Michael G. McDonald, 2000, MODFLOW-2000, The U.S. Geological Survey Modular Ground-Water Model-User Guide to Modularization Concepts and the Ground-Water Flow Process, Open-File Report 00-92, U.S. Geological Survey (U.S.G.S)

Haykin, S, 2008, Neural Networks and Learning Machines ( $3^{\text {rd }}$ Edition), pp.936, Prentice Hall, ISBN-10: 0131471392, ISBN-13: 978-0131471399

Heidarzadeh, M., Muhari, A. and Wijanarto, A. B., 2019, Insights on the Source of the 28 September 2018 Sulawesi Tsunami, Indonesia Based on Spectral Analyses and Numerical Simulations, Pure and Applied Geophysics, Vol. 176, 25-43, doi: 10.1007/s00024-018-2065-9

Hemmerich, J., Asilar, E. and Ecker, G. F., 2020, COVER: conformational oversampling as data augmentation for molecules, Journal of Cheminformatics, Vol.12, 18, doi: 10.1186/s13321-020-00420-z

Hernández-Pajares, M., Juan, J. M., Sanz, J., Orus, R., Garcia-Rigo, A., Feltens J., Komjathy A., Schaer S. C. and Krankowski, A., 2009, The IGS VTEC maps: a reliable source of ionospheric information since 1998, Journal of Geodesy, 83, 263-275, doi: 10.1007/s00190-008-0266-1.

Heidarzadeh, M., Harada, T., Satake, K., Ishibe, T. and Takagawa, 2017, Tsunamis from strike-slip earthquakes in the Wharton Basin, northeast Indian Ocean: March 2016 Mw7.8 event and its relationship with the April $2012 \mathrm{Mw} 8.6$ event, Geophysical Journal International, Vol.211(3), 1601-1612, doi: $10.1093 / \mathrm{gji} / \mathrm{ggx} 395$

Hines, C. O., 1960, Internal Atmospheric Gravity Waves at Ionospheric Heights, Canadian Journal of Physics, Vol. 38 (11), 1441-1481, doi: 10.1139/p60-150

Hines, C. O., 1972, Gravity Waves in the Atmosphere, Nature, Vol. 239, 73-78, doi: 10.1038/239073a0

Hofmann-Wellenhof, B., Lichtenegger, H. and Collins, J., 2001, Global Positioning System: Theory and Practice, pp.382, Springer-Verlag Wien, ISBN: 978-3-211-83534-0, doi: 10.1007/978-3-7091-6199-9

Kamogawa, M., Orihara, Y., Tsurudome, C., Tomida, Y., Kanaya, T., Ikeda, D., Gusman, A. R., Kakinami, Y., Liu, J. Y. and Toyoda, A., 2016, A possible space-based tsunami early warning system using observations of the tsunami ionospheric hole, Scientific Reports, Vol. 6, Article number, 37989, doi: 10.1038/srep37989

Kherani, E. A., Lognonné, P., Hébert, H., Rolland, L., Astafyeva, E., Occhipinti, G., Coïsson, P., Walwer, D. and de Paula, E. R., 2012, Modelling of the total electronic content and magnetic field anomalies generated by the 2011 Tohoku-Oki tsunami and associated acoustic-gravity waves, Geophysical Journal International, Vol.191 (3), 1049-1066, doi: 10.1111/j.1365-246X.2012.05617.x

Koundal, D. and Gupta, S., 2020, Advances in Computational Techniques for Biomedical Image Analysis, Academic Press, pp.322, ISBN: 978-0-12-820024-7, doi: 10.1016/C2019-0-00819-9

Lean, J. L., Meier, R.R., Picone, J. M., Sassi, F., Emmert, J. T., and Richards, P. G., 2016, Ionospheric total electron content: Spatial patterns of variability, Journal of Geophysical Research, Space Physics, Vol.121 (10), 367-10,402, doi: 10.1002/2016JA023210

Leble, S. and Smirnova, E., 2019, Tsunami-Launched Acoustic Wave in the Layered Atmosphere: Explicit Formulas Including Electron Density Disturbances, Atmosphere, 10(10), 629, doi: 10.3390/atmos 10100629

Kawashima, I. and Toh, H., 2016, Tsunami-generated magnetic fields may constrain focal mechanisms of earthquakes, Scientific Reports, Vol. 6, Article number, 28603, doi:10.1038/srep28603

Kong, H., L. Wang, E. K. Teoh, X. Li., J. G. Wang, R. Venkateswarlu, 2005, Generalized 2D principal component analysis for face image representation and recognition. Neural Networks, 18 (5-6), 585-594, doi: 10.1016/j.neunet.2005.06.041

Lilley, F. E. M., White, A. and Heinson, G. S., 2001, Earth's magnetic field: ocean current contributions to vertical profiles in deep oceans, Geophysical Journal International, Vol. 147(1), 163-175, doi: 10.1046/j.1365-246X.2001.00514.X

Lin, J.W. 2015, Early warning of tsunami from seismo-ionospheric fluctuation after Japan's March 11, 
2011, M=9.0 Tohoku earthquake using two-dimensional principal component analysis, Annals of Geophysics, Vol.58 (4), A0442, doi:10.4401/ag-6710

Lin, J. W., J. S., Chiou and C. T., Chao, 2018, Determining Neuronal Number in Each Hidden Layer Using Earthquake Catalogues as Training Data in Training an Embedded Back Propagation Neural Network for Predicting Earthquake Magnitude, IEEE Access, Vol. 6, 52582 - 52597, doi: 10.1109/ACCESS.2018.2870189

Lin, J.W., and J. S. Chiou, 2019, Active Probability Backpropagation Neural Network Model for Monthly Prediction of Probabilistic Seismic Hazard Analysis in Taiwan, IEEE Access, Vol.7, 108990-109014, Doi: 10.1109/ACCESS.2019.2932991

Lin, J. W. and J. S. Chiou, 2020, Detecting Total Electron Content Precursors Before Earthquakes by Examining Total Electron Content Images Based on Butterworth Filter in Convolutional Neural Networks, IEEE Access, Vol.8, 110478 - 110494, doi: 10.1109/ACCESS.2020.3001337

Lin, S. C., Wu, T. R., Yen E., Chen, H. Y., Hsu, J., Tsai, Y. L., Lee. C. J., and Liu, P. L. F., 2015, Development of a tsunami early warning system for the South China Sea, Ocean Engineering, Vol. 100, 1-18, doi: 10.1016/j.oceaneng.2015.02.003

Liu, J. Y., C. H. Chen, C. H. Lin, H. F. Tsai, C. H. Chen, and M. Kamogawa, 2011, Ionospheric disturbances triggered by the 11 March 2011 M9.0 Tohoku earthquake, J. Geophys. Res., 116, A06319, doi: 10.1029/2011JA016761

Liu, J. Y., Lin, C. Y., Tsai, Y L., Liu, T. C., Hattori, K., Sun, Y. Y. and Wu, T. R., 2019, Ionospheric GNSS Total Electron Content for Tsunami Warning, Journal of Earthquake and Tsunami, Vol. 13 (05n06), 1941007, doi: 10.1142/S1793431119410070

Maleki E. and Zabihollah, A., 2016, Modeling of shot peening effects on the surface properties of A (TiB + $\mathrm{TiC}) / \mathrm{Ti}-6 \mathrm{Al}-4 \mathrm{~V}$ composite employing arterial neural networks, Materials and technology, Vol. 50 (6), 851-860, doi: 10.17222/mit.2015.140.

Meng, X., Komjathy, A., Verkhoglyadova, O. P., Yang, Y. M., Deng, Y., and Mannucci, A. J., 2015, A new physics-based modeling approach for tsunami-ionosphere coupling, Geophysical Research Letters, Vol.42 (12), 4736-4744, doi: 10.1002/2015GL064610

Minami, T., Schnepf, N. R. and Toh, H., 2021, Tsunami-generated magnetic fields have primary and secondary arrivals like seismic waves, Scientific Reports, Vol. 11, 2287, doi: $10.1038 / \mathrm{s} 41598-021-81820-5$

Mohamed, Z. E., 2019, Using the artificial neural networks for prediction and validating solar radiation, Journal of the Egyptian Mathematical Society, Vol. 27, 47, doi; 10.1186/s42787-019-0043-8

Molchanov, O. A., and Hayakawa, M., 1998, Subionospheric VLF signal disturbances possibly related to earthquakes, Journal of Geophysical Research, Space Physics, Vol.103 (A8), 17489-17504, doi: 10.1029/98JA00999

Nguyen, D., and Widrow, B. 2009, Improving the learning speed of 2-layer neural networks by choosing initial values of the adaptive weights. Stanford University, Stanford, CA: Information Systems Laboratory

Occhipinti, G., Kherani, E. A. and Lognonné, P., 2008, Geomagnetic dependence of ionospheric disturbances induced by tsunamigenic internal gravity waves, Geophysical Journal International, Vol. 173 (3), 753-765, doi: 10.1111/j.1365-246X.2008.03760.x

Occhipinti, G., Coisson, P., Makela, J. J., Allgeyer, S., Kherani, A., Hébert, H., Lognonné, P., 2011, Three-dimensional numerical modeling of tsunami-related internal gravity waves in the Hawaiian atmosphere, Earth Planets Space, Vol. 63 (7), 847-851, doi: 10.5047/eps.2011.06.051

Occhipinti, G., 2015, The Seismology of the Planet Mongo: The 2015 Ionospheric Seismology Review, Subduction Dynamics: From Mantle Flow to Mega Disasters, Geophysical Monograph Series, Vol. 211, 169-182, doi: 10.1002/9781118888865.ch9

Okal, E. A. and Synolakis, 2016, Sequencing of tsunami waves: why the first wave is not always the largest, Geophysical Journal International, Vol.204 (2), 719-735, doi: 10.1093/gji/ggv457

Omatu, S., Rodríguez, S., Villarrubia, G., Faria, P., Sitek, P. and Prieto, J., 2018, Distributed Computing and Artificial Intelligence, 14th International Conference, Springer International Publishing, Germany, pp.344, ISBN: 978-3-319-62410-5, doi: 10.1007/978-3-319-62410-5

Palm, R. B., 2012, Prediction as a candidate for learning deep hierarchical models of data, Master's thesis, Technical University of Denmark, DTU Informatics, Denmark, pp. 87

Pennin, S., 2018, Tsunami Waves, Undergraduate Journal of Mathematical Modeling: One + Two: Vol. 8 
Pasini, A., 2015, Artificial neural networks for small dataset analysis, Journal of Thoracic Disease, Vol. 7(5), 953-960, doi: 10.3978/j.issn.2072-1439.2015.04.61

Ravanelli, M., Occhipinti, G., Savastano, G., Komjathy, A., Shume, E. B. and Crespi, M., 2021, GNSS total variometric approach: first demonstration of a tool for real-time tsunami genesis estimation, Scientific Reports, Vol. 11, 3114, doi: 10.1038/s41598-021-82532-6

Ren, X., Zhang, X., Xie, W., Zhang, K., Yuan, Y. and Li, X, 2016, Global Ionospheric Modelling using Multi-GNSS: BeiDou, Galileo, GLONASS and GPS, Scientific Reports, Vol. 6, 33499, doi: 10.1038/srep33499

Rolland, L. M., Occhipinti, G., Lognonné, P. and Loevenbruck, A., 2010, Ionospheric gravity waves detected offshore Hawaii after tsunamis, Geophysical Research Letters, Vol.37 (17), L17101, doi: 10.1029/2010GL044479

Šachl, L., Martinec, Z., Velímský, J., Irrgang, C., Petereit, J., Saynisch, J., Einšpigel, D. and Schnepf, N. R., 2019, Modelling of electromagnetic signatures of global ocean circulation: physical approximations and numerical issues, Earth, Planets and Space, Vol. 71, 58, doi: 10.1186/s40623-019-1033-7

Seddiki, K., Saudemont, P., Precioso F., Ogrinc, N., Salzet, M., Fournier, I. and Droit, A, 2020, Cumulative learning enables convolutional neural network representations for small mass spectrometry data classification, Nature Communications, Vol. 11, 5595, doi: 10.1038/s41467-020-19354-z.

Shaikhina, T. and Khovanova, N. A., 2017, Handling limited datasets with neural networks in medicalapplications: A small-data approach, Artificial Intelligence in Medicine, Vol. 75, 51-63, doi: 10.1016/j.artmed.2016.12.003

Shi, C. and Wei, N., 2020, Satellite Navigation for Digital Earth, Manual of Digital Earth, 125-160, Springer, Singapore, ISBN: 978-981-32-9915-3, doi:10.1007/978-981-32-9915-3_4

Shinagawa, H., Iyemori, T., Saito, S., and Maruyama, T., 2007, A numerical simulation of ionospheric and atmospheric variations associated with the Sumatra earthquake on December 26, 2004. Earth, Planets Space, Vol. 59 (9), 1015-1026, doi: 10.1186/BF03352042

Sim, S. Y. and Huang, Z, 2016, An Experimental Study of Tsunami Amplification by a Coastal Cliff, Journal of Coastal Research, Vol. 32 (3), 611-618, doi: 10.2112/JCOASTRES-D-15-00032.1

Sorokin, V.M. and Yaschenko, A. K., 2021, Generation of magnetic field in the low-latitude ionosphere by tsunami wave, Journal of Atmospheric and Solar-Terrestrial Physics, Vol. 213, 105521, doi: 10.1016/j.jastp.2020.105521

Sun, L., Wan, W., Ding, F., and Mao, T., 2007, Gravity wave propagation in the realistic atmosphere based on a three-dimensional transfer function model, Annales Geophysicae, Vol. 25 (9), 1979-1986, doi: 10.5194/angeo-25-1979-2007

Sun, Y. S., Chen, P. F., Chen, C. C., Lee, Y. T., Ma, K. F. and Wu, T.R., 2018, Assessment of the peak tsunami amplitude associated with a large earthquake occurring along the southernmost Ryukyu subduction zone in the region of Taiwan, Natural Hazards and Earth System Science, Vol.18, 2081-2092, doi: 10.5194/nhess-18-2081-2018

Tuntisukrarom, K. and R. Cheerarot, 2020, Prediction of Compressive Strength Behavior of Ground Bottom Ash Concrete by an Artificial Neural Network, Vol. 2020, Article ID 2608231, doi: $10.1155 / 2020 / 2608231$

Widiyantoro, S, Gunawan, E., Muhari, A., Rawlinson, N., Mori, J., Hanifa, N. R., Susilo, S, Supendi, P., Shiddiqi, H. A., Nugraha, A. D. and H. E. Putra, H. E., 2020, Implications for megathrust earthquakes and tsunamis from seismic gaps south of Java Indonesia, Scientific Reports, Vol. 10, Article number, 15274, doi: 10.1038/s41598-020-72142-z

Xu, Z. H., Du, C. P. and Xia, M. Y., 2018, Electromagnetic Fields Due to the Wake of a Moving Slender Body in a Finite-Depth Ocean with Density Stratification, Scientific Reports, Vol. 8, 14647, doi: 10.1038/s41598-018-32789-1

Yang, J., D. Zhang, A.F. Frangi, and J.Y Yang, 2004, Two-dimensional PCA: a new approach to appearance-based face representation and recognition. IEEE Transactions on Pattern analysis and Machine Intelligence, Vol. 26 (1), 131-137, Doi: 10.1109/TPAMI.2004.1261097

Yu, Y., Wang, W., and Hickey, M. P., 2017, Ionospheric signatures of gravity waves produced by the 2004 Sumatra and 2011 Tohoku tsunamis: A modeling study, Journal of Geophysical Research, Space Physics, Vol.122 (1), 1146-1162, doi: 10.1002/2016JA023116

Zakeri, Z., Mansfield, N., Sunderland, C., and Omurtag, A., 2020, Cross-validating models of continuous data from simulation and experiment by using linear regression and artificial neural networks, 


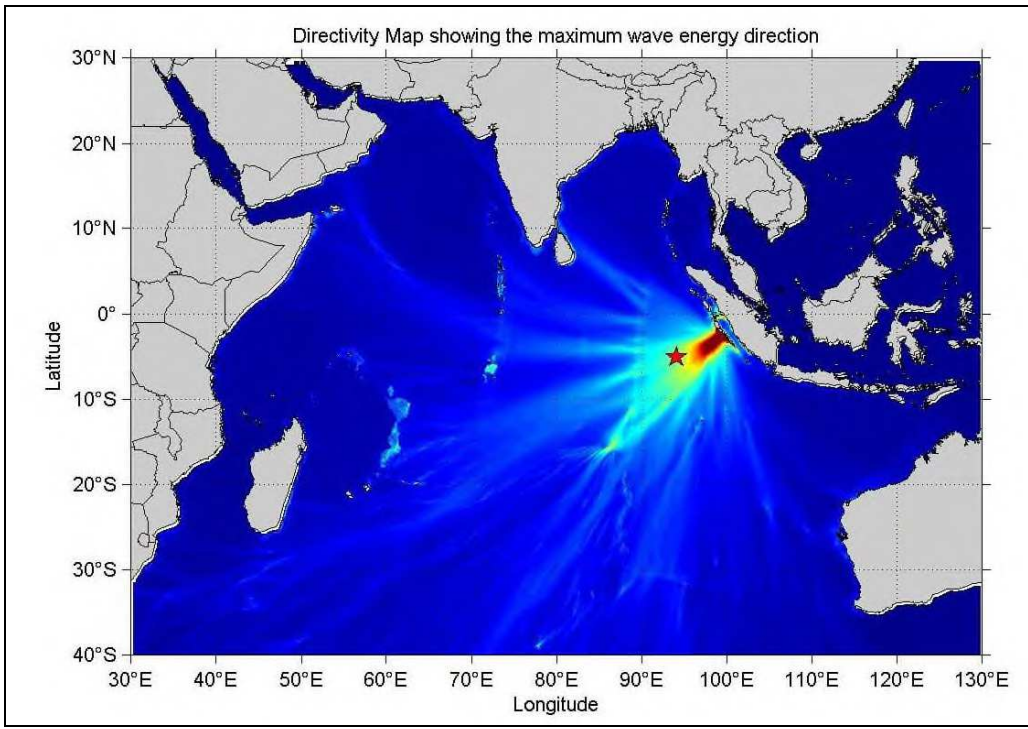

Fig. 1. Arrival of the tsunami at the station $\left(10.40^{\circ} \mathrm{S}, 105.67^{\circ} \mathrm{E}\right)$ at 15.02 on March 2, 2016 (UTC) (the red star indicates location of this station). The estimated amplitude was $0.11 \mathrm{~m}$ (represented by the long red strip) around Christmas Island (International Tsunami Information Center, ITIC; Indian Tsunami Early Warning Centre, ITEWC).

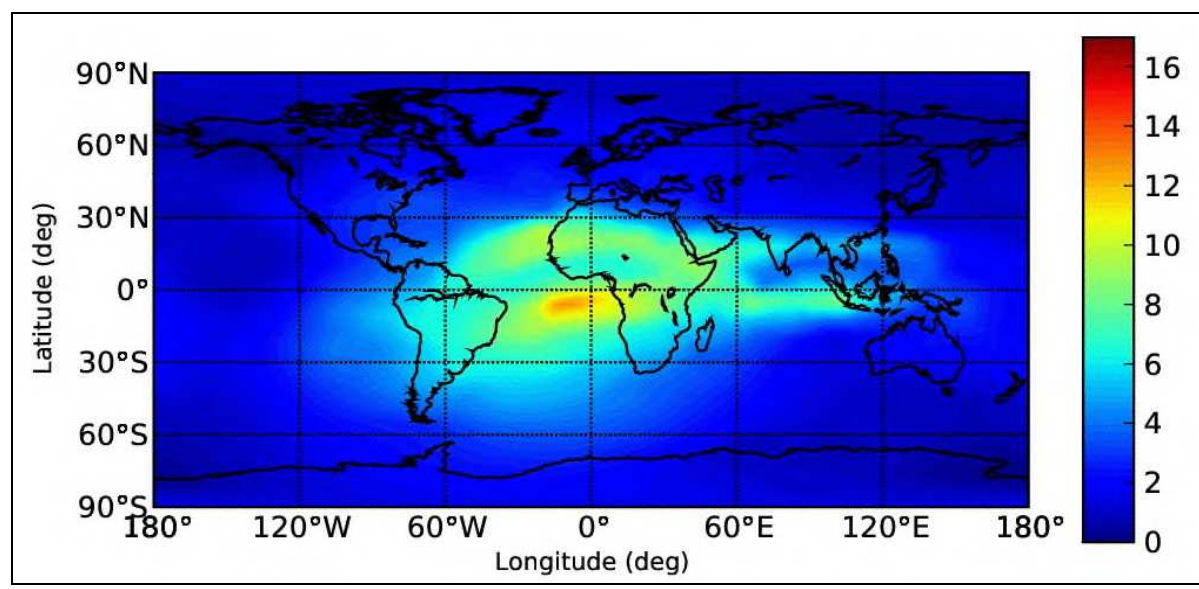

Fig. 2. Global ionospheric total electron content (TEC) map (GIM) at 13:20 on March 2, 2016 (UTC). The unit of TEC is TECu, and $1 \mathrm{TECu}=10^{16} \mathrm{e} / \mathrm{m}^{2}$; the symbols "e" and " $\mathrm{m}$ " are electrons and meters, respectively (Hofmann-Wellenhof et al., 2001). The traveling ionospheric disturbances (TIDs) are not easy to determine from the increasing or decreasing TECu in this TEC map (Source: GDGPS system). 
First Principal Eigenvalues (13:20 UTC)(B2DPCA)

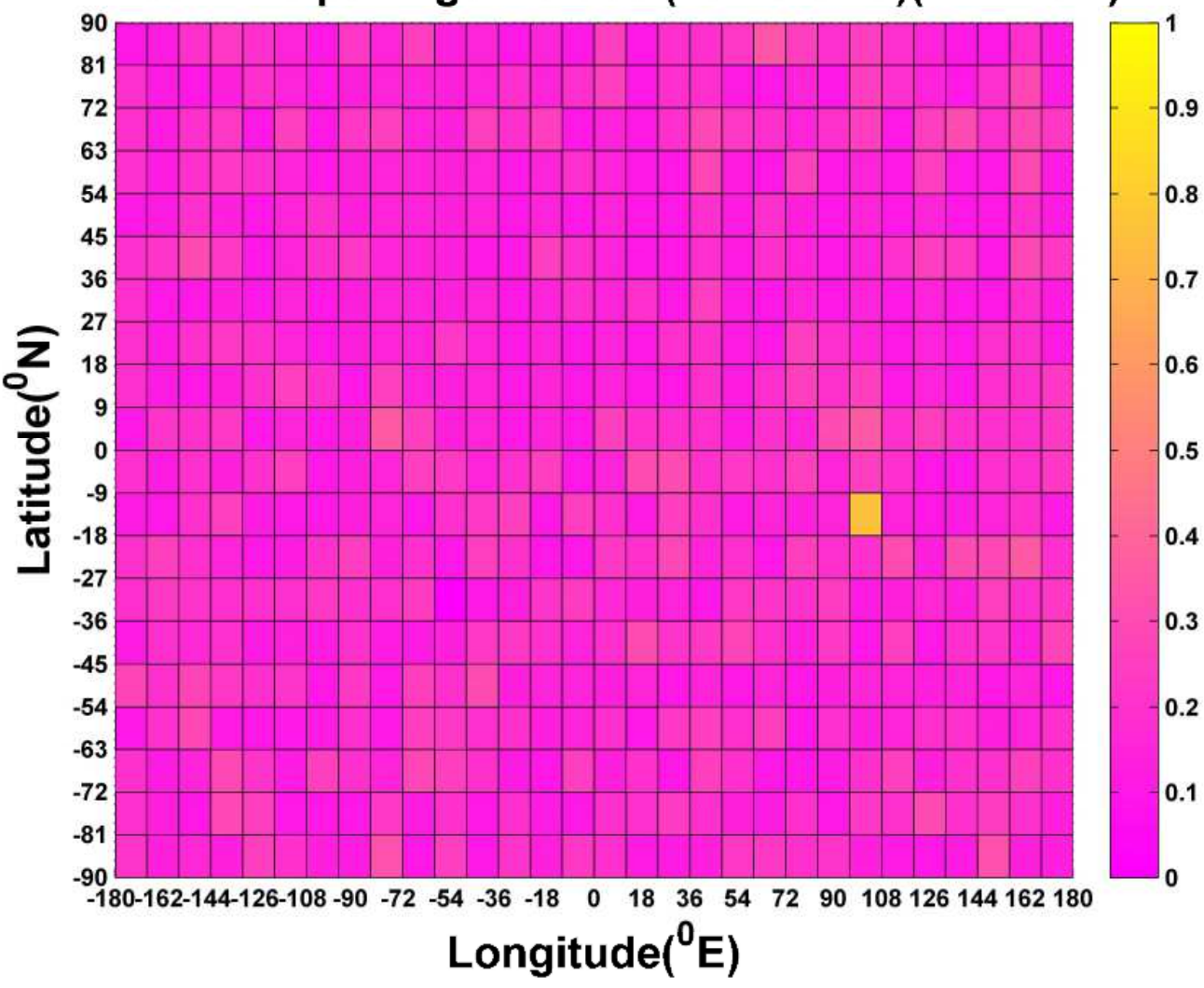

(a) 


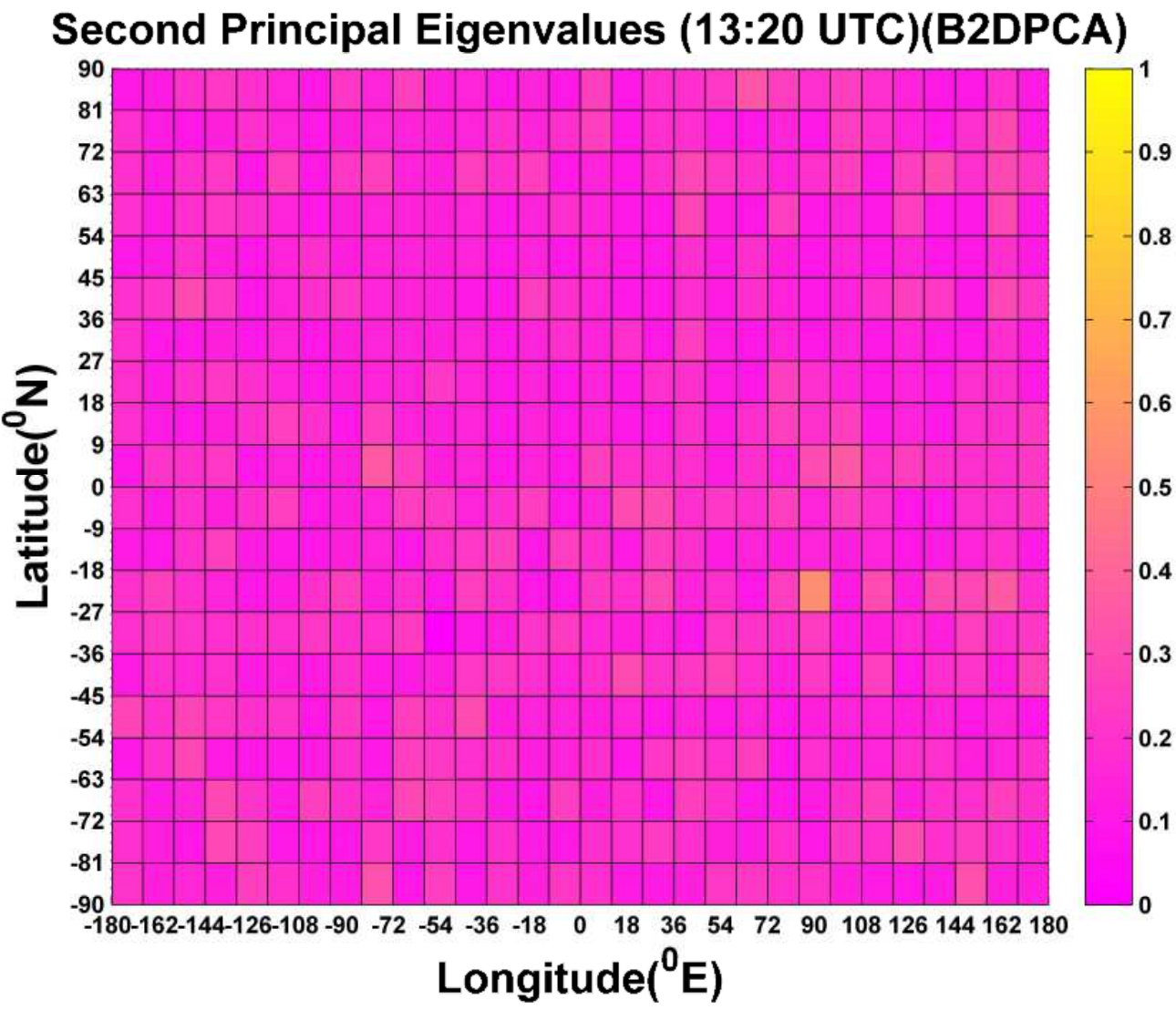

(b)

Fig. 3. (a) Magnitudes of first principal eigenvalues and (b) magnitudes of second principal eigenvalues corresponding to Fig. 2 obtained using bilateral projection-based two-dimensional principal component analysis (B2DPCA). 
First Principal Eigenvalues (13:20 UTC)(PCA)

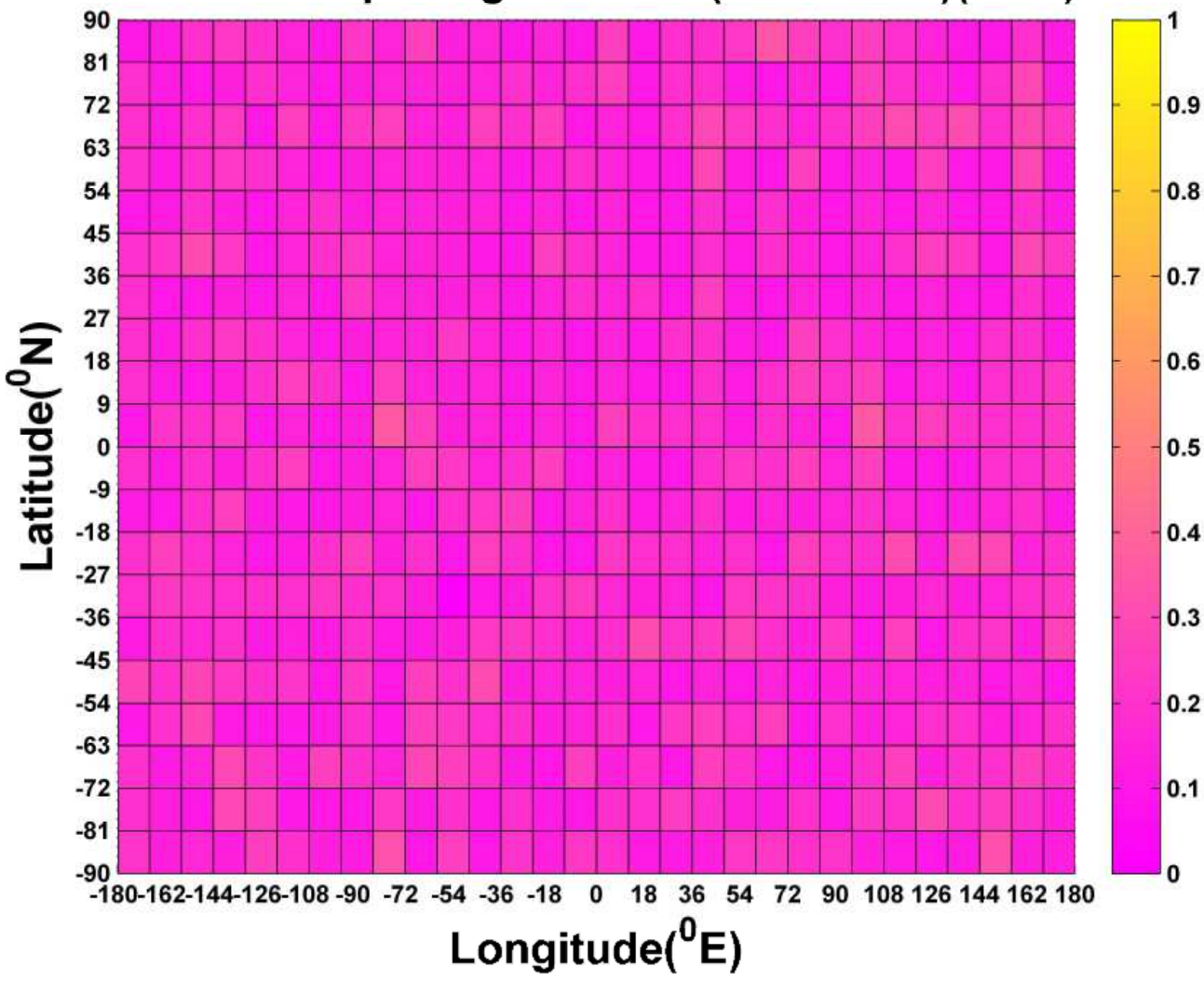




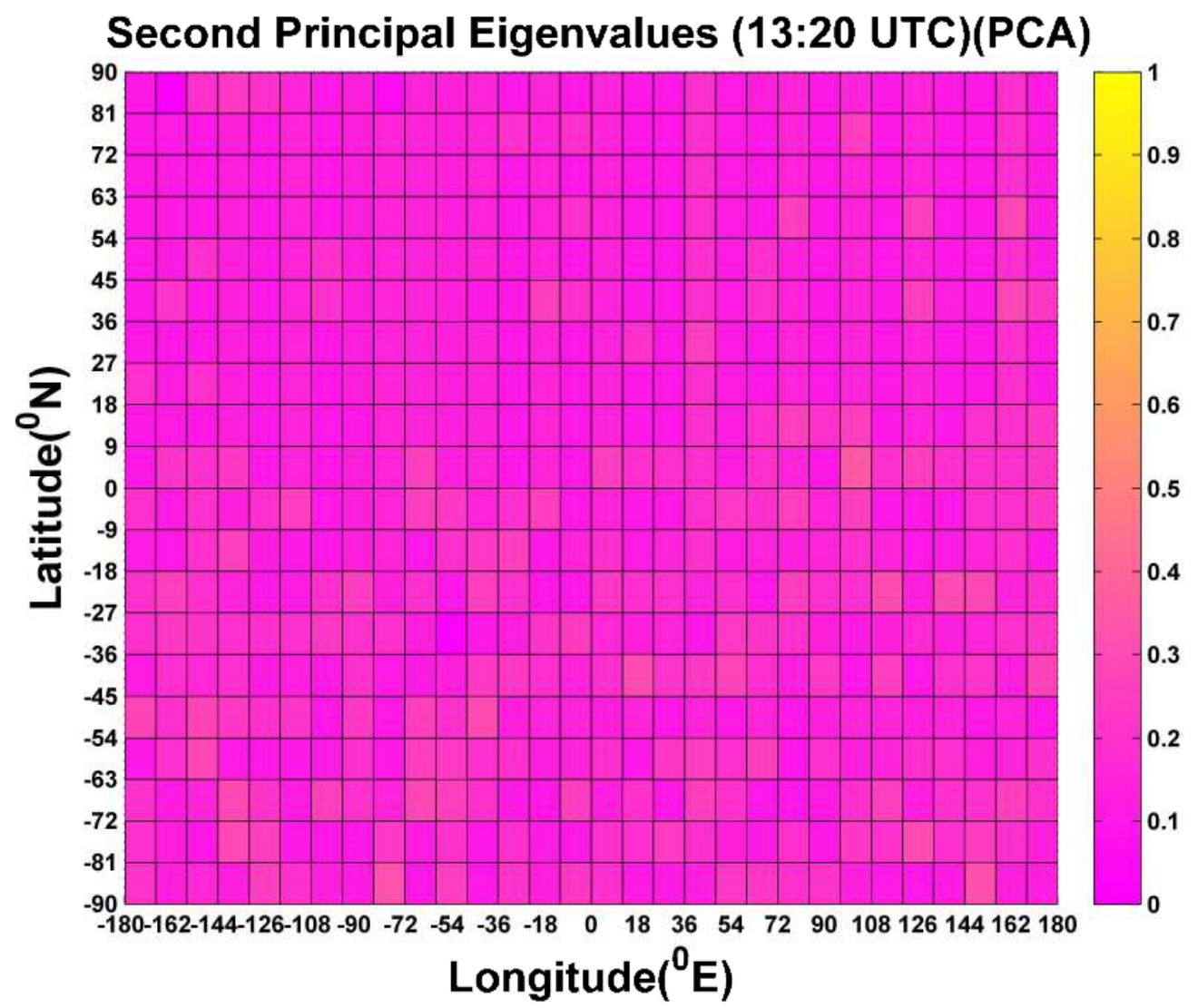

(b)

Fig. 4. (a) Magnitudes of first principal eigenvalues and (b) magnitudes of the second principal eigenvalues corresponding to Fig. 2 obtained using principal component analysis (PCA).

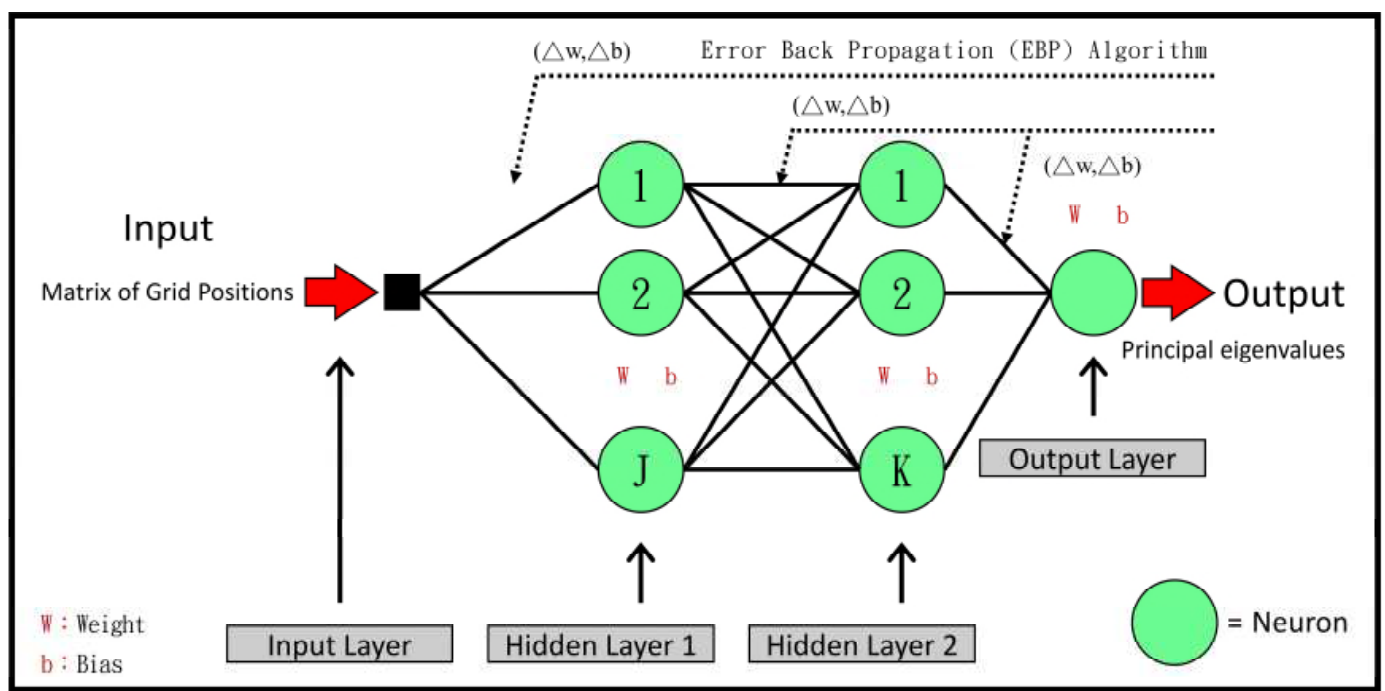

Fig. 5. Framework of a four-layer back-propagation neural network (BPNN) with two hidden layers. $J$ and $K$ are the number of neurons in the first and second hidden layers (Hidden Layers 1 and 2), respectively. A grid indicates a principal eigenvalue in Fig. 3 and 4. For the training input, for example, the position of a grid was at a latitude of $81^{\circ}$ to $90^{\circ}\left({ }^{\circ} \mathrm{N}\right)$ and a longitude of $-180^{\circ}$ to $-168^{\circ}\left({ }^{\circ} \mathrm{E}\right)$, and then this 
position is defined as $\mathrm{p}(1,1)$, which was an element of the input matrix called the Matrix of Grid Positions (note: the size of each grid was $9^{\circ}$ latitude $\times 12^{\circ}$ longitude). The output was a temporary output in each epoch of the training process using the error backpropagation (EBP) algorithm (Omatu et al., 2018). The principal eigenvalues are also called target outputs (Tuntisukrarom and Cheerarot, 2020), which are the real principal eigenvalues presented in Fig. 3.

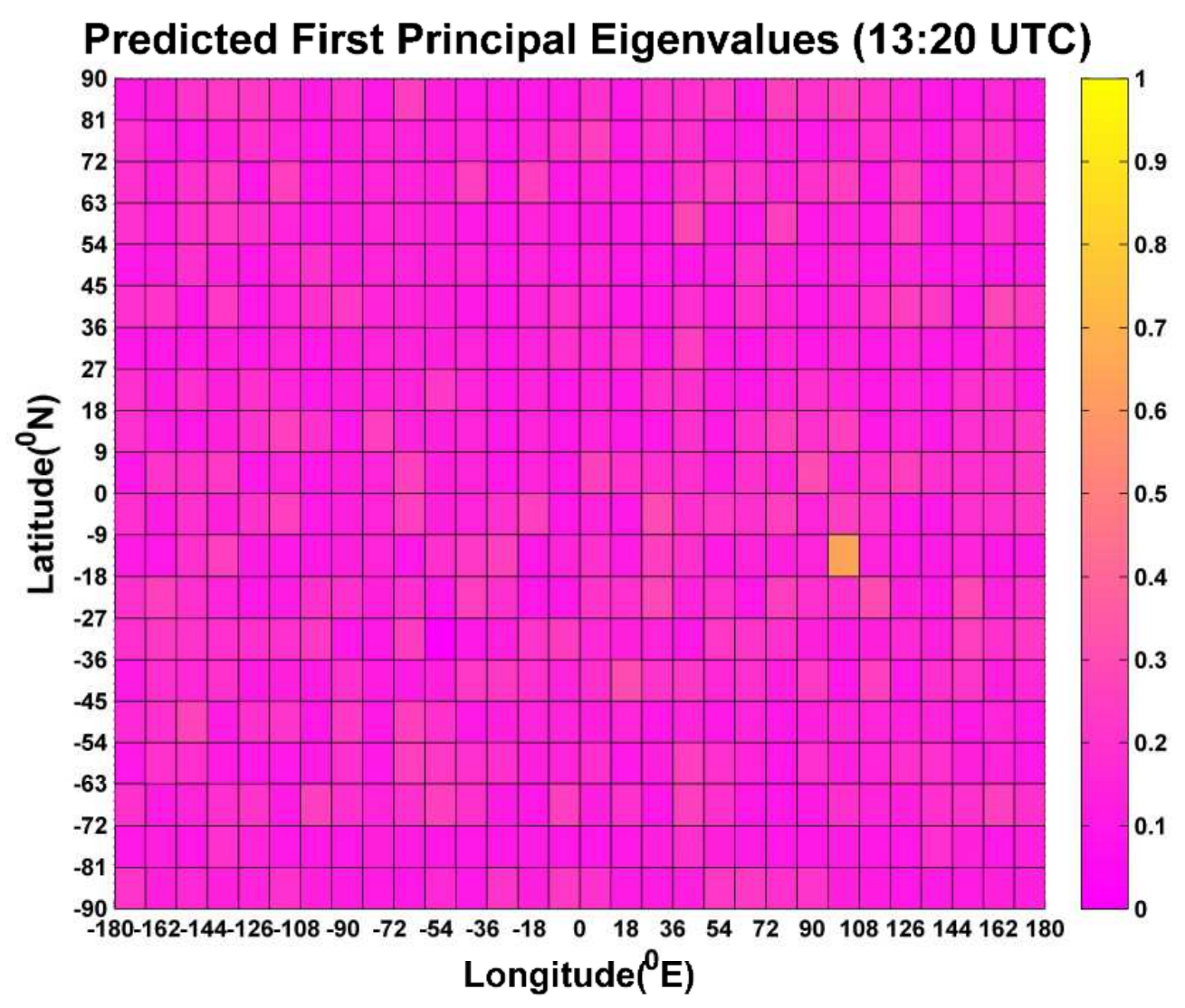

(a) 


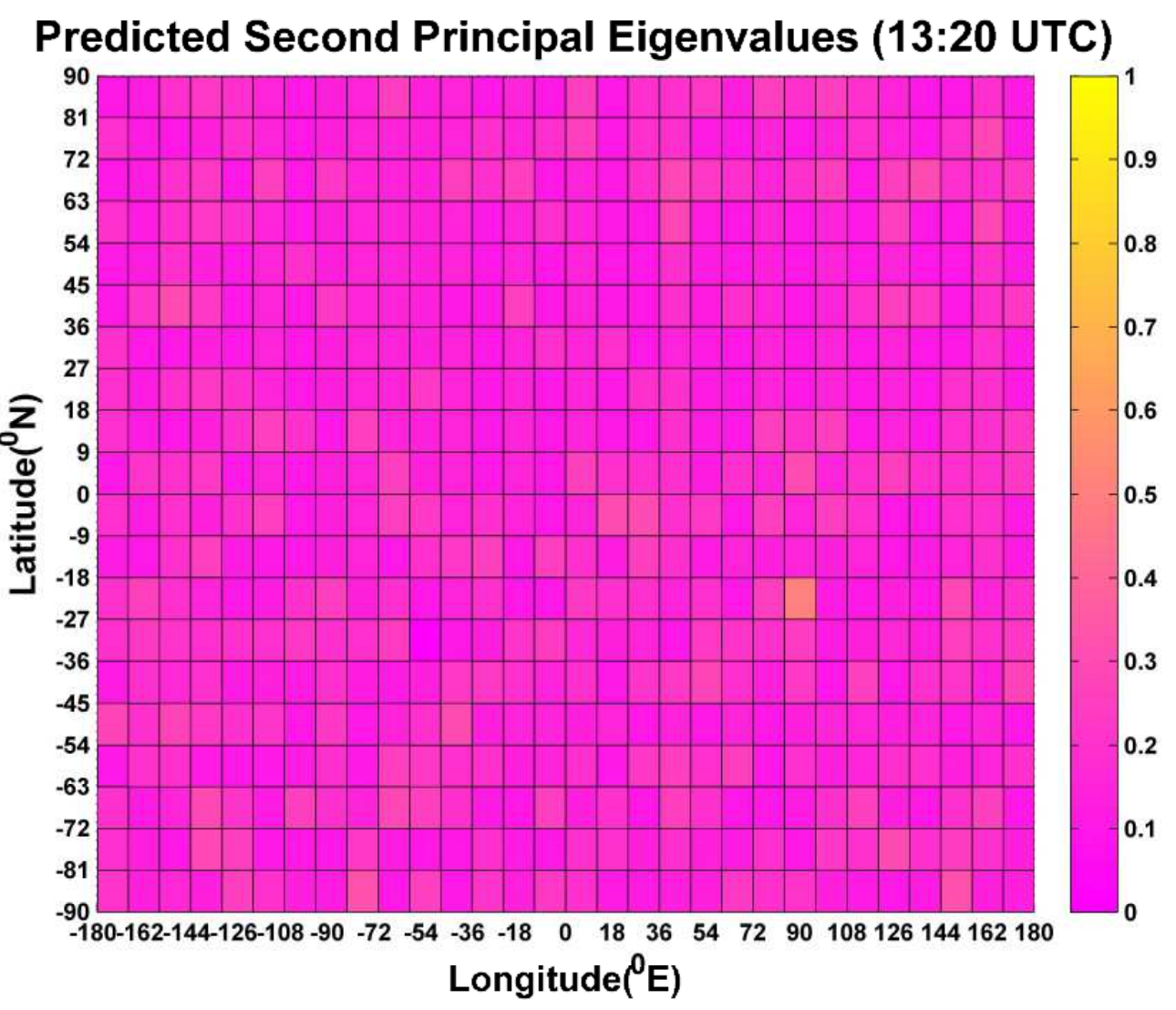

(b)

Fig. 6. (a) Predicted magnitudes of first principal eigenvalues and (b) predicted magnitudes of second principal eigenvalues, both corresponding to Fig. 3, obtained using the first BPNN model. 


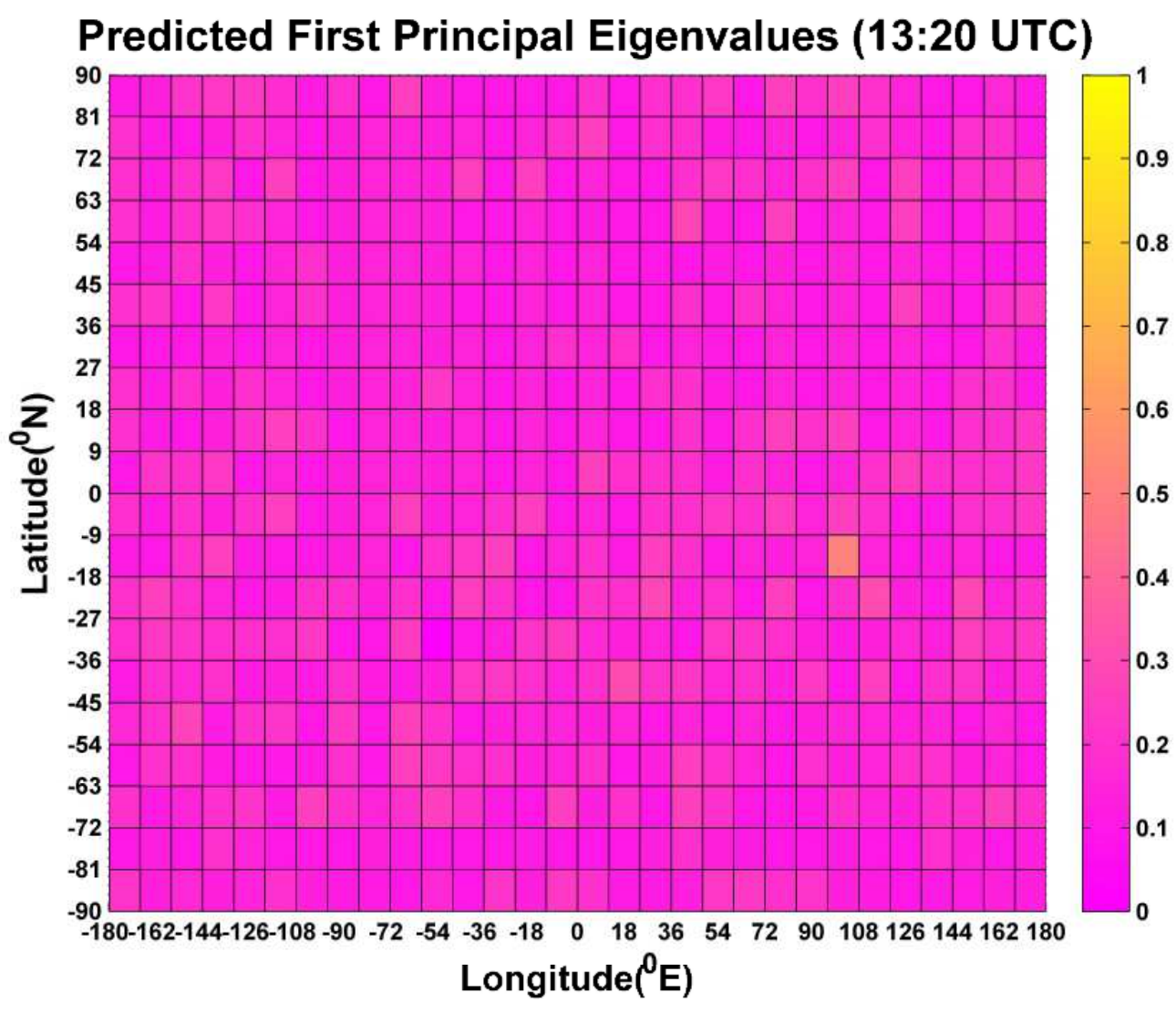




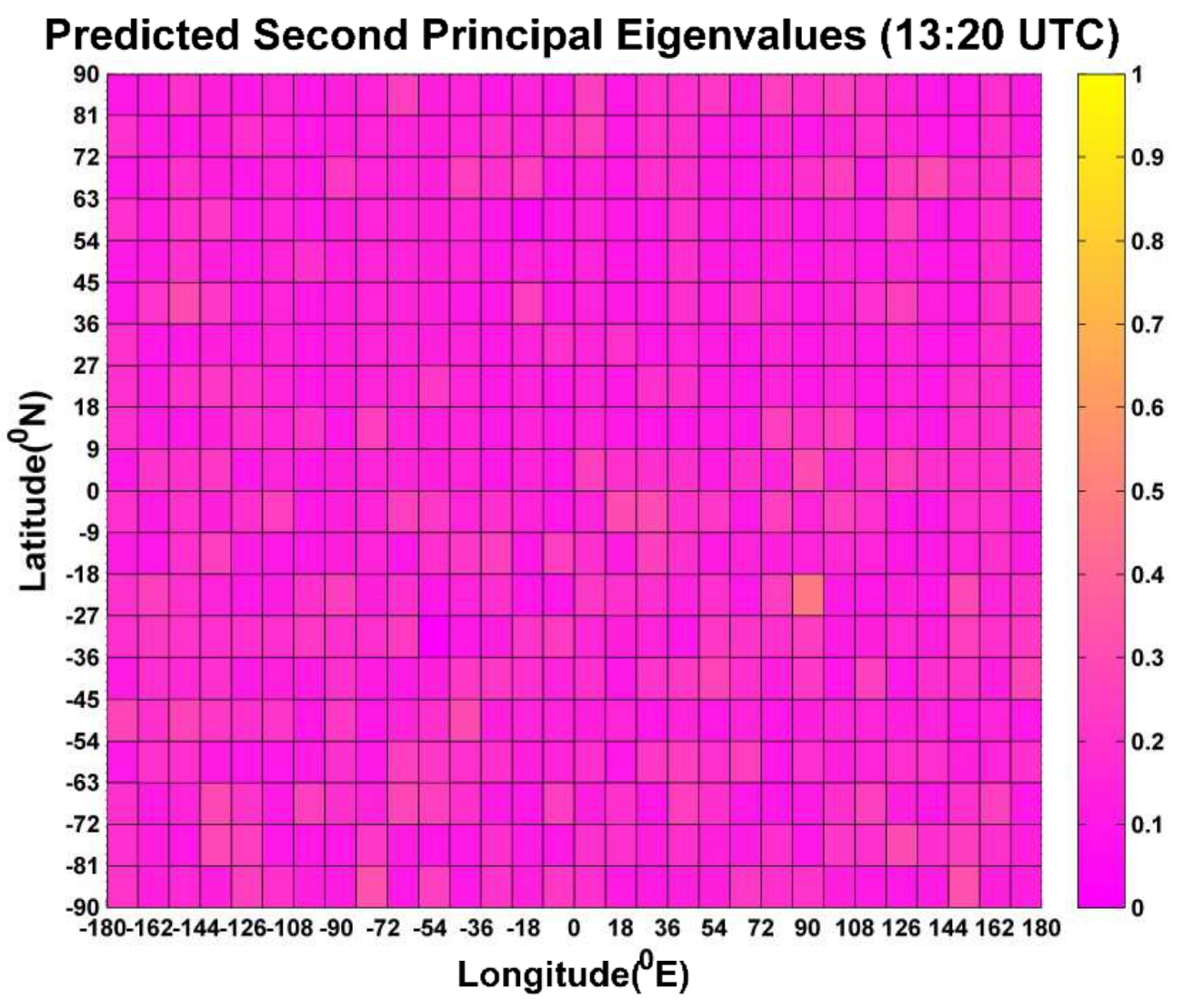

763 Fig. 7. Predicted magnitudes of (a) first and (b) second principal eigenvalues, both 764 765 766 
Determining the framework of BPNN Model

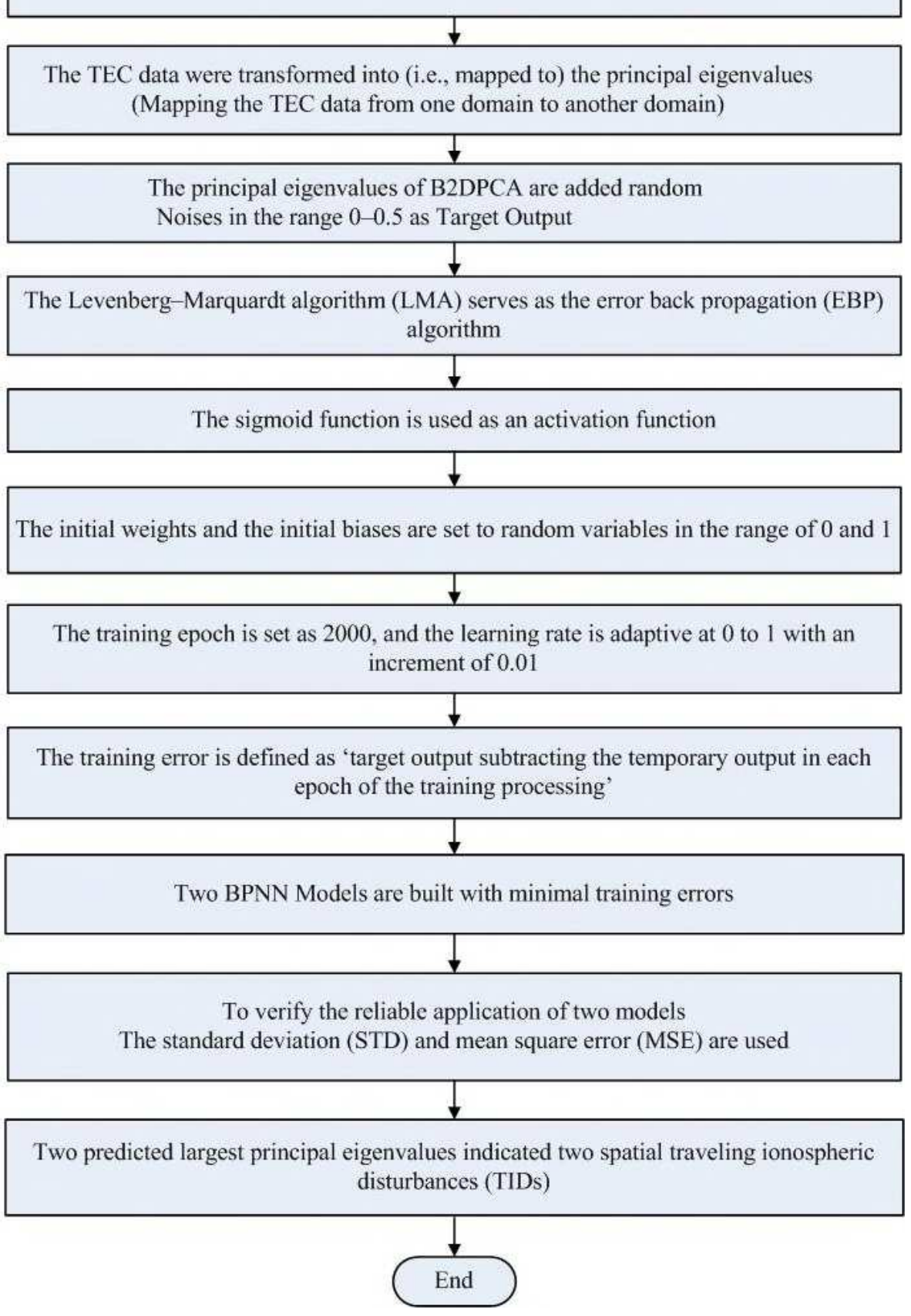

Fig. 8. Flowchart for creating two BPNN models. 


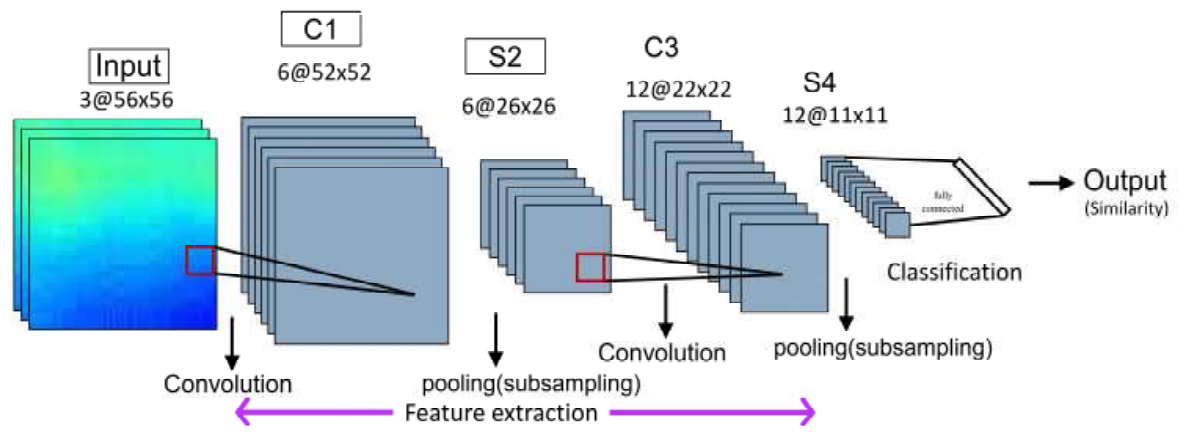

Fig. 9. Framework of two-dimensional convolutional neural networks (CNNs) with two hidden layers. For example, the symbol "3@" means three training images (Input). Pooling is an operation for subsampling feature maps to reduce the computing time. The training images from the global TEC map, depicted in Fig. 2 as inputs, are classified to identify TIDs using the algorithm called fully connected in a fully connected layer (similarity as Output). The output is a temporary output in each epoch of the training process using the EBP algorithm (Omatu et al., 2018).

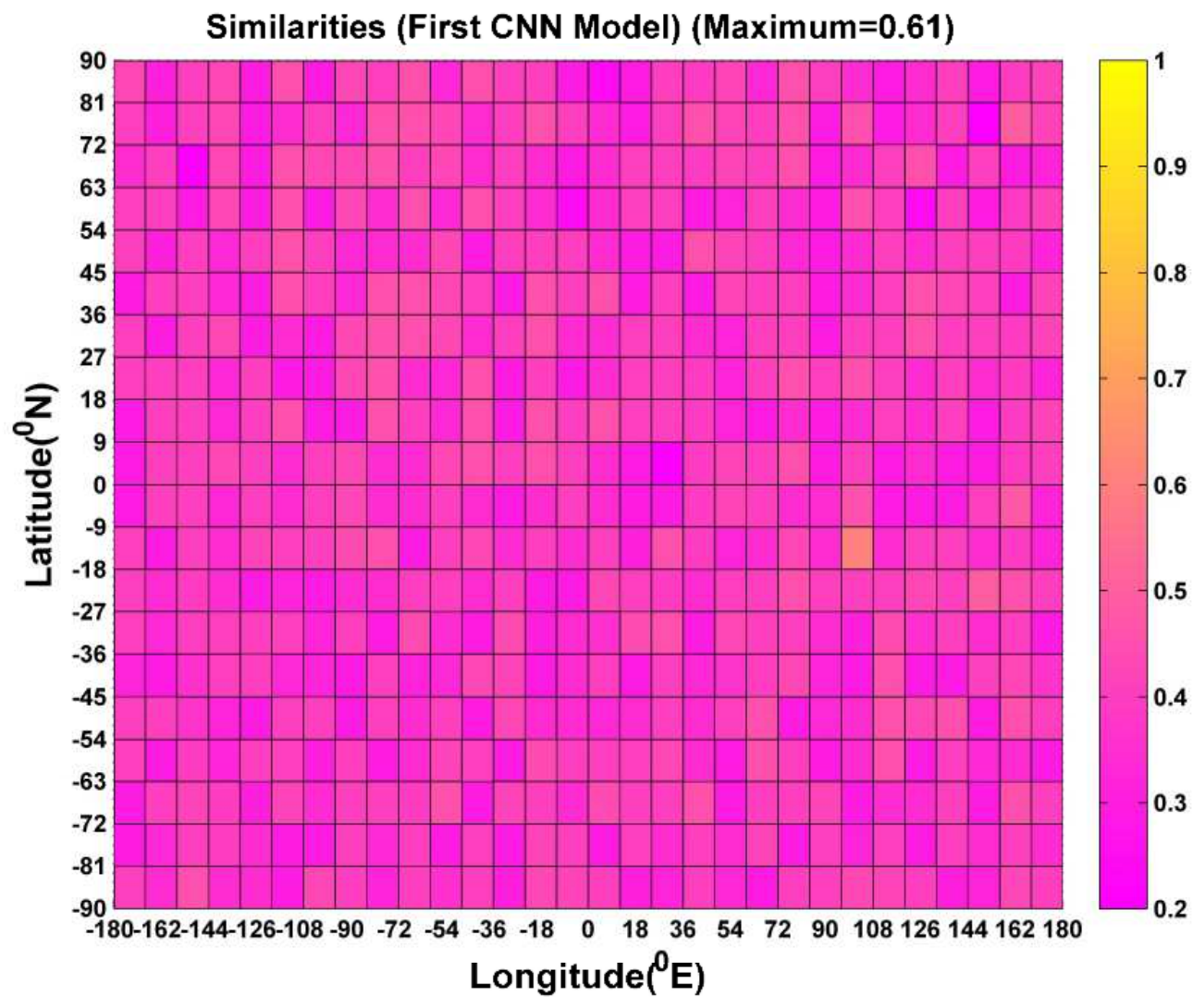

Fig. 10. Similarities for the grids with first principal eigenvalues corresponding to those in Fig. 3(a) based on the first CNN model. The maximum value was 0.61 , and other values are $<0.5$. The image was classified as the second classification, and they were located at the same positions as the largest first principal eigenvalues in Fig. 3(a). Therefore, the image corresponds to a TID. 


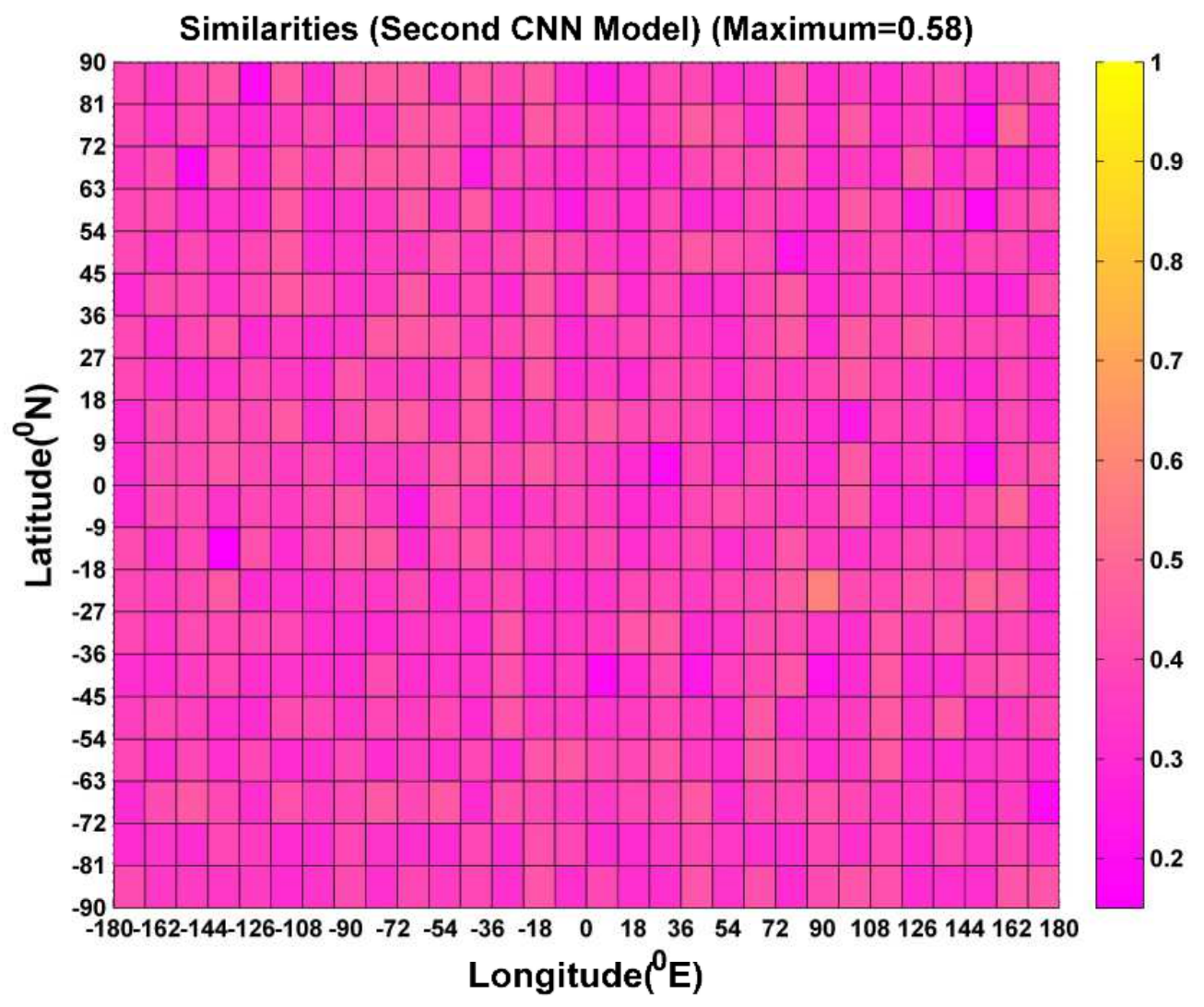

Fig. 11. Similarities for the grids with the second principal eigenvalues corresponding to Fig. 3(b) based on the second CNN model. The maximum value was 0.58 , and other values were $<0.5$. The image was classified as the second classification, and they were located at the same positions as the largest second 791 principal eigenvalues in Fig. 3(b). Therefore, the image corresponds to a TID. 
Determining the framework of CNN Model with

Two hidden layers. Each hidden layer has 10 Neurons

Spilt 600 images from Figure 2 as Training

images (called Training Inputs) and similarities of images with 0 and 1 as Target Outputs

1

A low pass filter called a Butterworth filter is applied to reduce wedge effects for each split images

This Butterworth filter is applied to remove the black curves and lines, which indicate contours of lands and scales of the latitude and longitude, respectively

Levenberg-Marquardt algorithm (LMA) served as the error back propagation (EBP) algorithm

(1)

The training epoch is set as 2000 and the batch size is 3

Learning rate is adaptive in the range at $0-1$ with an increment of 0.01

The initial weights and biases are set to random variables

Normalized training data in the range at $0-1$ with feature scaling

The training error is defined as 'target output subtracting the temporary output in each epoch of the training processing'

The two CNN models are bulit with minimal training errors

The predicted error is defined as 'predicted value subtracting the target output'

To verify and evaluate the reliable application and the prediction accuracy of of two models with the standard deviation (STD) and mean square error (MSE)

This previous verifying and evaluating are a cross validation

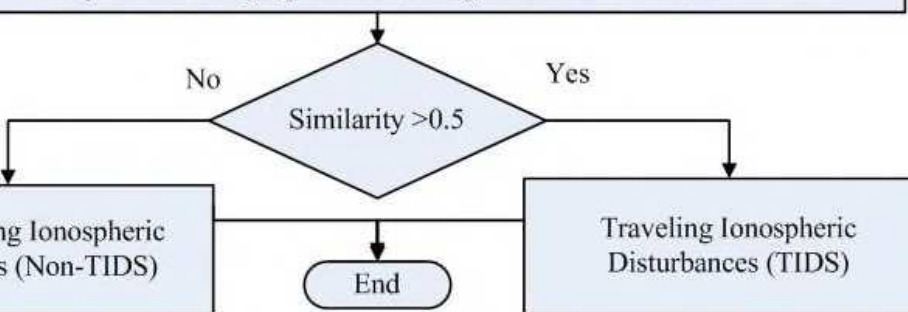

Non-Traveling lonospheri
Disturbances (Non-TIDS)

Fig. 12. Flowchart for creating the two CNN models. 


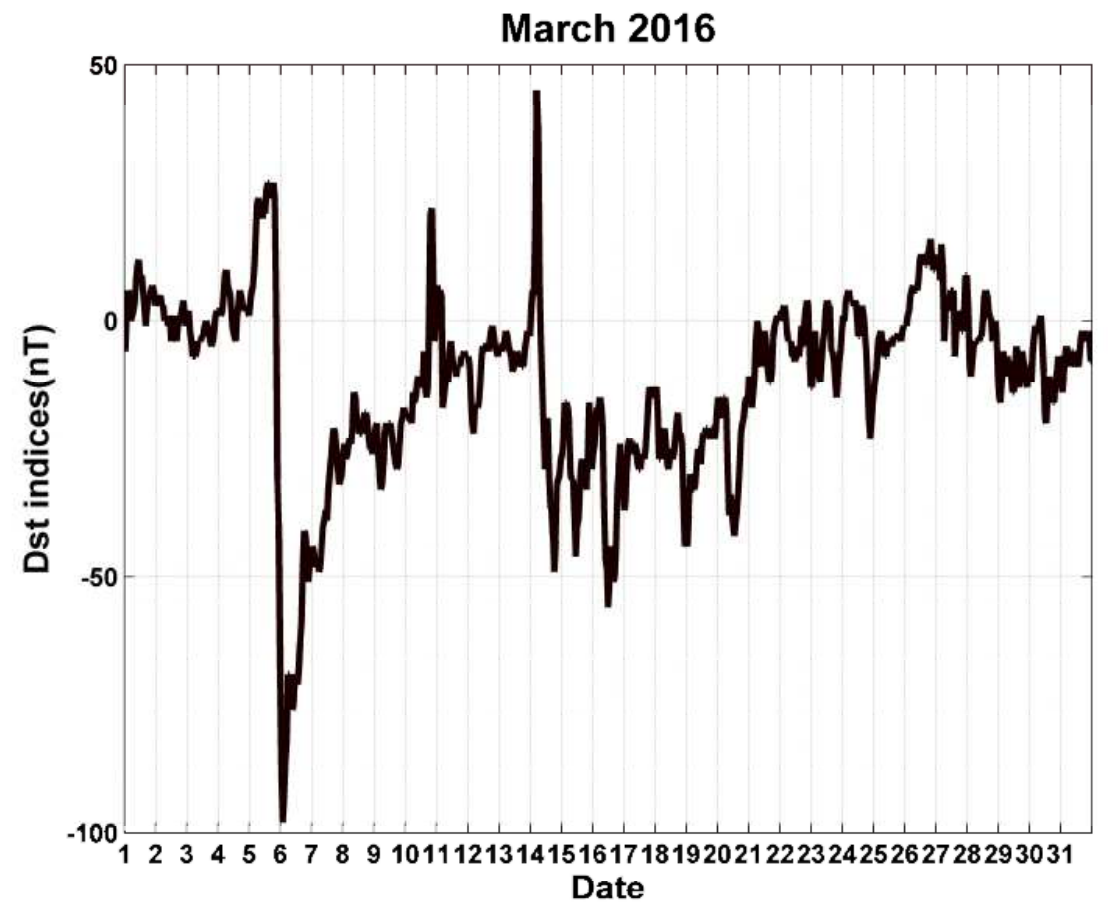

Fig. 13. Disturbance storm time (Dst) indices on March 2016 (unit: nT, sampling rate: per hour) (UTC) (World Data Centre for Geomagnetism, Kyoto, Japan). The horizontal timescale from 2 to 3 indicates the variations of the Dst indices on March 2, 2016 as an example.

801 